hep-ph/0611194

\title{
Determining Neutrino and Supernova Parameters with a Galactic Supernova
}

\author{
Solveig Skadhauge and Renata Zukanovich Funchal \\ Instituto de Física, Universidade de São Paulo, \\ C. P. 66.318, 05315-970 São Paulo, SP, Brazil
}

\begin{abstract}
We investigate the sensitivity of some of the proposed next-generation neutrino experiments to a galactic supernova. In particular, we study how well the supernova parameters (the average energies and luminosities) can be separated from the unknown neutrino oscillation parameters $\left(\theta_{13}\right.$ and the neutrino mass hierarchy). Three types of experiments, all in the 100 kilo-ton class, are compared. These are: a 540 kton water-Cherenkov detector, a 100 kton liquid Argon detector and a 50 kton scintillator detector. We demonstrate that practically all of these proposed detectors have the possibility to determine the hierarchy of the neutrino masses if the angle $\theta_{13}$ is sufficiently large $\left(\sin ^{2}\left(\theta_{13}\right) \gtrsim 10^{-4}\right)$ and the hierarchy of the average energies is larger than about $20 \%$. They can at the same time determine some of the supernova parameters well. The average energy of the $\nu_{\mu}$ and $\nu_{\tau}$ species can be determined within $5 \%$ uncertainty in most of the parameter space suggested by supernova simulations. The detection of several separable channels measuring different combinations of charged current and neutral current processes is crucial for determining the value of $\theta_{13}$ and the hierarchy. However, there are cases where a few of the SN parameters can be determined rather well even if only the main charged current detection channel is available.
\end{abstract}

PACS numbers: 14.60.Pq,25.30.Pt,97.60.Bw

Typeset by REVTEX 


\section{INTRODUCTION}

The fortuitous observation of a handful of neutrinos from the supernova 1987A [1, 2] started the era of experimental supernova (SN) neutrino physics. It confirmed our main ideas of the physics of a supernova from a core collapse, although also a set of (minor) disagreements were found. However, the low statistics of neutrino events collected from SN1987A, partially due to the fact that it happened at a distance of roughly $50 \mathrm{kpc}$ from Earth, was not sufficient to really extract much information. Evidently, with a larger detector and a closer by supernova, the prospects for gaining high accuracy information about both supernova and neutrino physics are immense. In the case of a galactic SN, one can observe of the order of 500 events per kton of detector material and there are several proposals for neutrino detectors in the 100 kton range [3].

A type II supernova is the death of a giant star and the huge emission of light as well as neutrinos is an effect of the gravitational core collapse of the star. In fact, about $99 \%$ of the total energy is emitted in the form of neutrinos and antineutrinos in a roughly 10 second interval. The consecutive burning of different elements, structured in an onion shell form, ends at the silicon burning phase. This phase produces an iron core and as fusion of iron is impossible, the gravitational collapse of the core is triggered once it reaches a well-known size, related to the Chandrasekhar limit. The core heats up during the implosion and photo-disintegration of the iron atoms starts, having as a byproduct free protons and neutrons. The subsequent neutronization gives rise to a $\nu_{e}$ flux from the deleptonization process $e+p \rightarrow \nu_{e}+n$. The $\mathrm{SN}$ densities in the interior of the core are so high that even neutrinos are trapped. However, neutrinos, interacting only weakly, manage to escape near the surface of the core region. A solid feature in all SN simulations is the formation of a shock wave caused by the rebounce of material falling on to the core. It is believed that this shock wave will cause the explosion of the outer material of the star. However, it has for a long time been a pending embarrassment, that SN simulations are incapable of producing the SN explosion as the shock wave looses too much energy to the media and halts before erupting the outer parts of the star. It has been suggested that neutrino physics might play a crucial role in re-energizing the shock wave [4], but until now a successful calculation has not been performed. After this so-called collapse phase, where a neutron star or, in rare cases, a black hole is formed, there is an accretion phase and a cooling phase.

In this article we will study the neutrino flux from the cooling phase. It is probably the phase where the physics is best controlled, although many things are still unknown. The energy spectra will be almost thermal, as the densities in the SN are so high that in fact the neutrinos will be in thermal equilibrium. But what are the average energies of each neutrino 
flavor? Again, a robust feature seems to be the hierarchy of these average energies. Electron neutrinos can interact in the medium both through charged current (CC) and neutral current (NC) exchanges, whereas $\nu_{\mu}$ and $\nu_{\tau}$ suffer only NC interactions as there are no muons or taus in the SN material. As a consequence, electron neutrinos will maintain their thermal equilibrium to a larger radius, therefore escaping with a smaller temperature or, equivalently, lower average energy $\left\langle E_{e}\right\rangle$. Furthermore, as $\nu_{\mu}$ and $\nu_{\tau}$, both, only interact via NC, their energy spectra will have identical properties. We will treat them as indistinguishable, denoting them with the common index $x$. Anti-electron neutrinos also interact via $\mathrm{CC}$ reactions but with a smaller cross-section (since there are less protons than neutrons) resulting in a higher temperature (average energy $\left.\left\langle E_{\bar{e}}\right\rangle\right)$ than electron neutrinos. So, a hierarchy of the form $\left\langle E_{e}\right\rangle\left\langle\left\langle E_{\bar{e}}\right\rangle\left\langle\left\langle E_{x}\right\rangle\right.\right.$ is predicted to exist. However, the exact values of the average energies as well as the strength of the hierarchies, vary quite substantially in different simulations and also depend on the type of the progenitor star [5, 6].

Neutrino flavor transitions have been observed in atmospheric, solar, reactor and accelerator neutrino experiments. The simplest and most widely accepted way to explain these transitions is to allow neutrinos to have masses and mixings. Although, the neutrino oscillation parameters have been determined to increasingly astonishing precision during the past few years [7, 8, 9, 10, 11], currently,

$$
\begin{aligned}
+7.3 \times 10^{-5} \mathrm{eV}^{2} & <\Delta m_{21}^{2}<+9.0 \times 10^{-5} \mathrm{eV}^{2} \\
0.25 & <\sin ^{2} \theta_{12}<0.37 \\
1.5 \times 10^{-3} \mathrm{eV}^{2}<\left|\Delta m_{32}^{2}\right| & <3.4 \times 10^{-3} \mathrm{eV}^{2} \\
0.36 & <\sin ^{2} \theta_{23} \leq 0.64
\end{aligned}
$$

at $90 \% \mathrm{CL}$, some important points remain unknown. We still lack information on the absolute neutrino mass scale $m_{0}$, only an upper bound $m_{0} \lesssim 0.2-0.7 \mathrm{eV}$ [12] exist. The neutrino mass pattern is not yet completely established: we do not know if nature prefers the normal $\left(m_{3}>m_{2}>m_{1}\right)$ or the inverted $\left(m_{2}>m_{1}>m_{3}\right)$ mass hierarchy, where $m_{1}\left(m_{3}\right)$ is the mass of the neutrino state most (least) populated by the $\nu_{e}$ component. Moreover, we only have an upper limit on the mixing angle $\theta_{13}, \sin ^{2} \theta_{13} \lesssim 0.04$, given by the CHOOZ reactor experiment [13].

The supernova density profile is such that a neutrino oscillation resonance in the 31-channel, involving $\Delta m_{31}^{2}$ and $\theta_{13}$, is bound to happen. Whether this happens for neutrinos or antineutrinos depends on the neutrino mass hierarchy (if normal or inverted). Besides, the resonance is very sensitive to the parameter $\theta_{13}$. The value of the hopping probability changes from zero to one when $\theta_{13}$ goes from $10^{-4}$ to $10^{-1}$. Clearly, supernova neutrinos provide an excellent 
chance to determine two of the neutrino unknowns: the mass hierarchy and $\theta_{13}$. This fact has been pointed out and explored by several authors [14, 15, 16].

However, the open questions on the dynamics of the SN explosion could, in principle, plague the determination of neutrino properties. One might wonder if it will be possible to disentangle the uncertainties of the supernova physics from the uncertainties on the neutrino parameters, and use experimental measurements of the SN neutrino fluxes to extract new information on both, the SN explosion mechanism and the neutrino oscillation parameters.

In this article we analyze in detail the prospects for extracting the SN parameters as well as the neutrino oscillation parameters at three different types of next-generation detectors, from the measurements of neutrinos from the cooling phase of a galactic supernova. The most realistic next-generation experiments under present consideration are a megaton-scale water Cherenkov, a 100 kton liquid Argon and a 50 kton scintillation detector. We will study the performance of each of these detector types. In our analysis we will vary a total of seven parameters. Five are SN parameters: the average $\nu_{e}, \bar{\nu}_{e}$ and $\nu_{x}$ energies, respectively, $\left\langle E_{e}\right\rangle$, $\left\langle E_{\bar{e}}\right\rangle$ and $\left\langle E_{x}\right\rangle$; the ratio of the luminosities in $x$ and $e$ flavors, $\xi$ (we assume the $\bar{\nu}_{e}$ and $\nu_{e}$ luminosities to be equal) and finally the overall normalization of the fluxes, fixed by the total energy released $\left(E_{b}\right)$ and the distance to the exploding star $(D), E_{b} / D^{2}$. The last two are neutrino oscillation parameters: the value of the angle $\theta_{13}$ and the neutrino mass hierarchy. We will simulate an observed set of data for given values of these seven parameters and use a $\chi^{2}$ method to henceforth construct confidence levels for the determination of these unknowns. In particular, we perform a comparison, highlighting strengths and weaknesses of each type of proposed experiment. In this paper we only consider the performance of the detectors regarding supernova neutrinos. However, when making a decision of which detectors should be build, their sensitivity to many other processes, including nucleon decay, solar and atmospheric neutrinos, beta-beams and super-beams as well as a neutrino factory, should naturally also be analyzed [17].

There has been a number of earlier works on supernova neutrinos, with many papers discussing only the extraction of neutrino parameters. Refs. [18, 19], however, investigate the possibility to get information on SN physics from the SN neutrinos using Super-Kamiokande and SNO detectors. These analyses take into account fewer supernova parameters and only some of the detection channels (considered as inseparable) we will consider here. Also, the simultaneous analysis of both normal and inverted hierarchy was not performed. The analysis in Ref. [20] is very similar to the present study but concerns only a liquid Argon experiment. Bounds on neutrino masses, from the delayed time-of-flight as a function of the neutrino energy, obtainable with future large water Cherenkov as well as with a liquid scintillation experiment has also been discussed [21]. Other methods to extract information from a SN are: analyzing 
the earth matter effects [14, 22, 23, 24, 25, 26] which may occur if the supernova neutrinos traverse the Earth (mantle/core) before reaching the detector; studying the variation of particularly constructed variables [15, 27, 28], such as ratios of average energies, and recently the possibility of observing shock wave effects has attracted attention [15, 29, 30, 31, 32, 33].

It should be noted that the determination of the SN parameters from the other studies, such as Earth matter effects are difficult, without prior knowledge of the value of $\theta_{13}$. This is due to the fact that the Earth matter effects dependent on a combination of the hopping probability and the difference in unoscillated neutrino fluxes. Therefore, apparently a full analysis varying both neutrino and SN parameters is necessary for obtaining information on the SN parameters. In the present paper we will show to which maximal accuracy the SN parameters can be determined by the three types of experiments and how their determination depends on the values of the unknown neutrino parameters. Such a determination will likely be very helpful for the understanding of the physics of the core-collapse.

In the next section we present the parameterized neutrino flux from the SN cooling phase. In Sec. III we will discuss the analysis method and the three experimental setups we consider here. In Sec. IV we discuss our results and we devote the final section to our conclusions.

\section{THE NEUTRINO FLUX FROM THE COOLING PHASE}

In this analysis we will consider neutrinos emitted from the cooling phase of a type II supernova. This phase has the largest emission of energy with an expected total (time integrated) luminosity, $E_{b}$, of about $1-5 \times 10^{53} \mathrm{ergs}$. This luminosity is divided into all 6 flavors and we denote the individual contributions by $L_{i}$. We assume (as usual) that $\nu_{\mu}, \nu_{\tau}, \bar{\nu}_{\mu}, \bar{\nu}_{\tau}$ are indistinguishable ${ }^{\dagger}$ and denote them by the common index $x$. Thus, we have $E_{b}=L_{e}+L_{\bar{e}}+4 L_{x}$. We will furthermore assume that $\nu_{e}$ and $\bar{\nu}_{e}$ luminosities are identical, i.e., $L_{e}=L_{\bar{e}}$, which holds approximately in most SN simulation. We allow for a violation of luminosity equipartition by defining the parameter

$$
\xi=\frac{L_{x}}{L_{e}}
$$

In general, simulations compute a value of $\xi$ between 0.5 and 2 [5, 6].

We use the pinched Fermi-Dirac distribution for the energy-spectra of the neutrinos emitted from the supernova (the unoscillated flux)

$$
\phi_{i}^{0}(E)=\frac{1}{F_{3}\left(\eta_{i}\right) T_{i}^{4}} \frac{E^{2}}{\exp \left(E / T_{i}-\eta_{i}\right)+1} \quad, \quad i=e, \bar{e}, x
$$

\footnotetext{
$\dagger$ We neglect the small difference in $\nu_{i}$ and $\bar{\nu}_{i}, i=\mu, \tau$, fluxes originating from weak magnetism effects.
} 
where $F_{3}$ is a normalization function. The average energies, $\left\langle E_{i}\right\rangle$, are linearly related to the temperatures once the pinching parameter, $\eta_{i}$, is fixed. As explained in the introduction the supernova physics strongly suggests that these average energies follow a hierarchy; $\left\langle E_{e}\right\rangle<$ $\left\langle E_{\bar{e}}\right\rangle\left\langle\left\langle E_{x}\right\rangle\right.$. The SN simulations favor $0 \leq \eta_{e}, \eta_{\bar{e}} \leq 3$ and $\eta_{x}<2$. In our simulations we take all $\eta$ 's to be zero, corresponding to pure Fermi-Dirac spectra. This is a conservative choice, since superposition of narrower energy spectra will be easier for the detectors to distinguish. In the case the average energies vary with time, the value of $\eta$ will be smaller, thus justifying our choice. Naturally, it would be even better if one could vary also the pinching parameters, but this is beyond our scope.

The unoscillated $\nu_{i}$ flux at distance $D$ from the supernova is given by

$$
F_{\nu_{i}}^{0}=\frac{L_{i}}{4 \pi D^{2}} \phi_{i}^{0}(E)
$$

where all the luminosities are proportional to the total binding energy $E_{b}$. It is worth remembering that one will normally not be able to see an optical counterpart to the SN (if the SN is inline with the galactic center it will be obscured by dust), leaving the distance $D$ unknown. A crude estimate of the chance that an optical signal of the SN can be seen is only about one out of four. This should be compared to the fact that less than four supernovæ are expected in our galaxy per century. Therefore, in this article we will suppose that it is the combination $E_{b} / D^{2}$ that will be constrained by the SN neutrino detection. This can easily be translated to a constraint on the total emitted energy if the distance $D$ can be independently determined. Thus, the unoscillated flux of neutrinos from a supernova is parametrized by 5 variables: $\left\langle E_{e}\right\rangle$, $\left\langle E_{\bar{e}}\right\rangle,\left\langle E_{x}\right\rangle, \xi$ and $E_{b} / D^{2}$.

The flux produced in the interior of the star will change its flavor composition when traversing the outer parts of the star, due to neutrino flavor oscillations in matter. As we assume that the $\mu$ and $\tau$ fluxes are identical, the oscillated flux will only depend on the $\nu_{e}$ and $\bar{\nu}_{e}$ survival probabilities. These probabilities strongly depend on the unknown neutrino parameters: $\theta_{13}$ and the mass hierarchy. We will now summarize these effects assuming that the matter density of the SN scales as $\rho \sim r^{-3}$, which seems to be the most realistic density profile ${ }^{\S}$. We will use the approximation

$$
\rho(r)=C \cdot 10^{13}\left(\frac{10 \mathrm{~km}}{r}\right)^{3} \mathrm{~g} \cdot \mathrm{cm}^{-3},
$$

and we take the value $C=4$. Due to the mass gap $\left(\Delta m_{21}^{2} /\left|\Delta m_{32}^{2}\right| \approx 1 / 30\right)$ and the smallness of $\theta_{13}$, the dynamics of the $3-\nu$ system can be factorized as two $2-\nu$ sub-systems: a high $(\mathrm{H})$ one, driven by $\Delta m_{31}^{2}$ and $\theta_{13}$, and a low (L) one, driven by $\Delta m_{21}^{2}$ and $\theta_{12}$. Correspondently,

\footnotetext{
$\S$ For others density profiles see 34].
} 

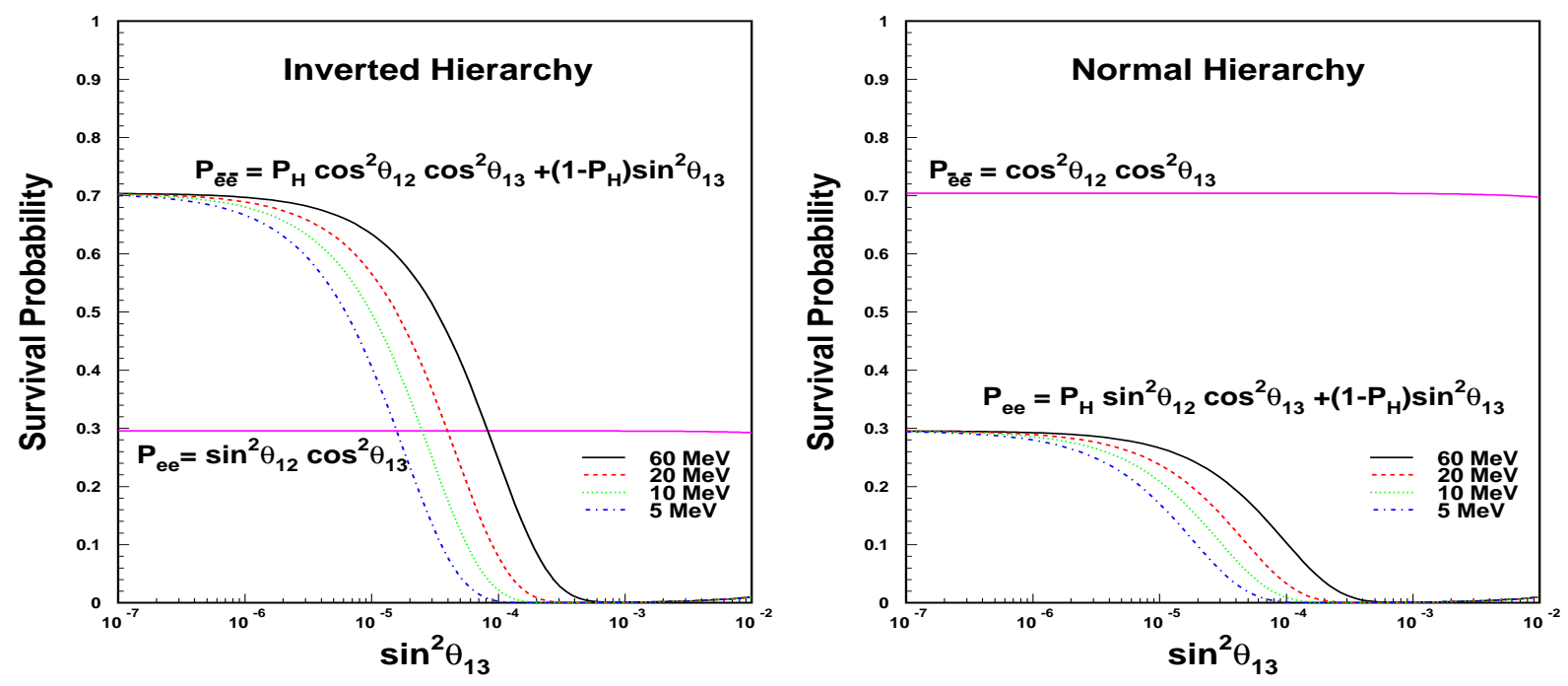

FIG. 1: The $\nu_{e}, P_{e e}$, and $\bar{\nu}_{e}, P_{\bar{e} \bar{e}}$, survival probabilities as a functions of $\sin ^{2} \theta_{13}$ for four different neutrino energies. We show the cases of inverted (left panel) and normal (right panel) hierarchy. We have used $\sin ^{2} \theta_{12}=0.3$ and $\left|\Delta m_{31}^{2}\right|=3.0 \times 10^{-3} \mathrm{eV}^{2}$.

two resonances can happen as neutrinos travel through the SN. The H resonance will occur for neutrinos (antineutrinos) in the case of normal (inverted) hierarchy. In this case, there is a possible nonzero hopping probability $P_{H}$ for crossing between effective mass eigenstates. The L resonance will occur for neutrinos ${ }^{\ddagger}$ and due to the values of the solar parameters given in (1) it will be always adiabatic (the level crossing probability $P_{L} \approx 0$ ). Thus, we will not discuss this resonance further.

The hopping probability $P_{H}$ can be parametrized as

$$
P_{H}=\exp \left[-\sin ^{2} \theta_{13}\left(\frac{1.08 \cdot 10^{7}}{E}\right)^{2 / 3}\left(\frac{\left|\Delta m_{31}^{2}\right|}{10^{-3}}\right)^{2 / 3} C^{1 / 3}\right]
$$

where $E$ is in $\mathrm{MeV}$ and $\Delta m_{31}^{2}$ in units of $\mathrm{eV}^{2}{ }^{\dagger}$. It should be noticed that the value of $C$ (which is uncertain by about a factor of 4 ) affects the translation from $P_{H}$ to $\theta_{13}$. Therefore, depending on how well the value of $C$ can be determined, one might have to take this uncertainty into account when putting bounds on $\theta_{13}$. Knowing the type of the progenitor star would be a help in this case.

The $\nu_{e}$ and $\bar{\nu}_{e}$ survival probabilities, $P_{e e}$ and $P_{\bar{e} \bar{e}}$, respectively, are approximated for the

\footnotetext{
${ }^{\ddagger}$ Since SNO's demonstration that the solar CC/NC ratio is less than $\frac{1}{2}$ we know that $\Delta m_{21}^{2}>0$.

†Strictly speaking the $\Delta m^{2}$ in Eq. (77) should be $\Delta m_{32}^{2}$ for the normal hierarchy and $\Delta m_{31}^{2}$ for the inverted one. However since $\left|\Delta m_{31}^{2}\right| \approx\left|\Delta m_{32}^{2}\right|$, this is not relevant here.
} 
normal hierarchy by

$$
\begin{aligned}
& P_{e e} \simeq P_{H}\left|U_{e 2}\right|^{2}+\left(1-P_{H}\right)\left|U_{e 3}\right|^{2}, \\
& P_{\bar{e} \bar{e}} \simeq\left|U_{e 1}\right|^{2},
\end{aligned}
$$

where $U_{\alpha, i}, \alpha=e, \mu, \tau, i=1,2,3$ are the Maki-Nakagawa-Sakata neutrino mixing matrix elements and we have used here the standard parameterization. Using (2) we find $\left|U_{e 1}\right|^{2} \simeq 0.7$, $\left|U_{e 2}\right|^{2} \simeq 0.3$ and $\left|U_{e 3}\right|^{2}<10^{-2}$. And for the inverted hierarchy

$$
\begin{aligned}
& P_{e e} \simeq\left|U_{e 2}\right|^{2}, \\
& P_{\bar{e} \bar{e}} \simeq P_{H}\left|U_{e 1}\right|^{2}+\left(1-P_{H}\right)\left|U_{e 3}\right|^{2} .
\end{aligned}
$$

These survival probabilities as a function of $\sin ^{2} \theta_{13}$ are shown in Figure 1 for various neutrino energies and the two types of hierarchies. We will neglect collective flavor transformation of the neutrinos, which in a detailed analysis should be added to the conventional MSW conversion in the supernova environment [35].

The final fluxes arriving at Earth is simply given by

$$
\begin{aligned}
F_{\nu_{e}} & =F_{\nu_{e}}^{0} P_{e e}+F_{\nu_{x}}^{0}\left(1-P_{e e}\right), \\
F_{\bar{\nu}_{e}} & =F_{\bar{\nu}_{e}}^{0} P_{\bar{e} \bar{e}}+F_{\bar{\nu}_{x}}^{0}\left(1-P_{\bar{e} \bar{e}}\right), \\
F_{\nu_{\mu}}+F_{\nu_{\tau}} & =F_{\nu_{e}}^{0}\left(1-P_{e e}\right)+F_{\nu_{x}}^{0}\left(1+P_{e e}\right), \\
F_{\bar{\nu}_{\mu}}+F_{\bar{\nu}_{\tau}} & =F_{\bar{\nu}_{e}}^{0}\left(1-P_{\bar{e} \bar{e}}\right)+F_{\bar{\nu}_{x}}^{0}\left(1+P_{\bar{e} \bar{e}}\right) .
\end{aligned}
$$

Notice that in the case of luminosity equipartition and degeneracy in average energies, the flux is independent of the survival probabilities and thus also independent of the neutrino parameters.

When calculating the oscillation probabilities we have neglected the Earth matter effects [14]. These effects can be precisely calculated and thus will not affect our conclusions much, if the direction of the supernova is known. Even in the case that an optical counterpart of the SN can not be seen, the direction of the supernova can be determined rather well from the neutrino flux, if the detector (or another existing detector than the one being analyzed) can measure the elastic scattering of neutrinos on electrons. This detection channel is highly forward peaked and in the case of Hyper-Kamiokande the direction can be inferred to within $\sim 1^{\circ}$ [36]. Therefore, we will not take into account the Earth matter effects in our calculations. However, in section IV we will briefly discuss the possibility of extracting further information about the hierarchy by detecting Earth matter effects. 


\section{THE ANALYSIS METHOD AND THE DETECTORS}

In this section we will describe our method for studying the sensitivity of the detectors to the parameters under investigation of neutrinos from a nearby supernova. While we are rather optimistic in our choice of detector performances we will be taking somewhat difficult choices for the input SN parameters.

As explained earlier, the neutrino fluxes arriving at the detectors will depend on 7 parameters (5 SN dynamics parameters, and 2 neutrino physics parameters). For each experiment, we simulate the expected number of neutrino events at each observable mode, for a fixed set of these parameters. The artificially generated data will be our input data (the imagined true values $)$. We then construct a $\chi^{2}=\chi^{2}\left(\left\langle E_{e}\right\rangle,\left\langle E_{\bar{e}}\right\rangle,\left\langle E_{x}\right\rangle, \xi, E_{b} / D^{2}, \sin ^{2} \theta_{13}, \operatorname{sign}\left(\Delta m_{31}^{2}\right)\right)$ function in order to fit these unknown parameters to the input data in the usual way. We only consider statistical uncertainties in our $\chi^{2}$. This allows to compare the maximal attainable sensitivity for each detector type. We compute the allowed regions for each pair of unknown parameters to estimate the experimental sensitivity to them by marginalizing with respect to the other 4 parameters, for a fixed hierarchy. Since we construct the confidence level region for each hierarchy separately, our graphs will still be useful, in the case that the neutrino mass hierarchy is determined before a SN observation. Moreover, this allows us to conclude whether or not the hierarchy can be established.

The artificially generated data are constructed simply by calculating the theoretical expectation at the chosen set of input parameters. We have tested that this gives similar results as when a more realistic data set is used. We have constructed a Gaussian distributed data set, by choosing randomly a point from a Gaussian distribution centered around the expectation for the bin and of width equal to its square-root. There is no difference in the results from the Gaussian distributed data set and the data set given by the theoretical expectation, besides that the Gaussian data set might give a fluctuation of the allowed regions away from the central value. Generating the observed data set by the theoretical expectation allows for easy independent reconstruction and comparison and we therefore prefer to use this simpler method for generating the data.

We will in this analysis use the following parameters space when varying the SN parameters:

$$
\begin{aligned}
& \left\langle E_{e}\right\rangle \in[9-15] \mathrm{MeV}, \quad\left\langle\mathrm{E}_{\overline{\mathrm{e}}}\right\rangle \in[12-17] \mathrm{MeV}, \quad\left\langle\mathrm{E}_{\mathrm{x}}\right\rangle \in[15-30] \mathrm{MeV} \\
& \xi \in[0.5-2.0], \quad E_{b} / D^{2} \in(2.0-4.0) \times 10^{51} \mathrm{ergs} / \mathrm{kpc}^{2} .
\end{aligned}
$$

This parameter space is roughly what is expected from SN simulations [5, 6]. In any case if an analysis of SN neutrino observation cannot determine the parameters within the area suggested in (16), the SN simulations will probably do a better job. Naturally, the confidence levels and 
the accuracy with which each parameter can be measured, will depend on the input data. For instance, in the case when $\left\langle E_{e}\right\rangle=\left\langle E_{\bar{e}}\right\rangle=\left\langle E_{x}\right\rangle$ and $\xi=1$ the neutrino flux becomes independent of the survival probabilities and thus of the neutrino oscillation parameters. Therefore, in this special limit (luckily not favored from SN simulations) the neutrino parameters cannot be deduced from the observation of SN neutrinos. We will discuss how the neutrino parameter determination depend on the strength of the hierarchy of the average energies.

\begin{tabular}{|l||c|c|c|c|c|}
\hline & $\left\langle E_{e}\right\rangle(\mathrm{MeV})$ & $\left\langle E_{\bar{e}}\right\rangle(\mathrm{MeV})$ & $\left\langle E_{x}\right\rangle(\mathrm{MeV})$ & $\xi$ & $E_{b} / D^{2}\left(\mathrm{ergs} / \mathrm{kpc}^{2}\right)$ \\
\hline \hline point 1 & 12 & 15 & 18.0 & 1.50 & $3.0 \times 10^{51}$ \\
\hline point 2 & 12 & 15 & 18.0 & 0.75 & $3.0 \times 10^{51}$ \\
\hline point 3 & 12 & 15 & 16.5 & 1.50 & $3.0 \times 10^{51}$ \\
\hline
\end{tabular}

TABLE I: Definition of the input SN parameters at some reference points.

In Table I we define three points in the SN parameter space, that we will use as reference points. These points are situated within the expectation of the SN simulation given in equation 16. Most parameters are chosen at their central value. However, the value of $\xi$ has been chosen at two point slightly away from its central value (being 1), so as to see its impact on the accuracy with which the SN and neutrino parameters can be determined. Also the values of $\left\langle E_{x}\right\rangle$ is chosen at the lower range of its expectations. This has been done in order to see until which lower value a meaningful determination of the neutrino parameters can be expected. It is important to notice that increasing the value of $\left\langle E_{x}\right\rangle$ will increase the sensitivity of the detectors to the neutrino parameters as well as to the supernova parameters. First of all a higher value of $\left\langle E_{x}\right\rangle$ will give a larger number of events, since the detection cross-sections increases with energy. Also the increased hierarchy between $\left\langle E_{x}\right\rangle$ and $\left\langle E_{\bar{e}}\right\rangle$ will make the impact of the value of the survival probability larger. For points 1 and 2 we study a rather conservative case of a hierarchy of only $19 \%$ difference between $\left\langle E_{\bar{e}}\right\rangle$ and $\left\langle E_{x}\right\rangle$. For point 3 the hierarchy is of only $10 \%$, in which case it will become harder to determine the neutrino parameters. Although, such a weak hierarchy between the average energies seems rather unlikely it cannot yet be excluded.

The solar neutrino mixing angle and the atmospheric mass-square difference are fixed to values in the allowed region given in (2):

$$
\theta_{12}=0.575 \mathrm{rad}, \quad\left|\Delta m_{31}^{2}\right|=3.0 \times 10^{-3} \mathrm{eV}^{2}
$$

The atmospheric mixing angle does not enter into the calculation as $\nu_{\mu}$ and $\nu_{\tau}$ enter on the same footing both in the SN and in the detector (it introduces a unobservable rotation). Furthermore, as we do not take into account Earth matter effects, we have no dependence on the solar masssquare difference. When calculating the confidence levels we will just study the extreme cases 
for the true value of $\theta_{13}$. Meaning that we will only investigate two values of $P_{H}$; zero and one. In this case we have three scenarios for the input neutrino parameters:

1. Scenario i0: Inverted hierarchy and $P_{H} \simeq 0$ (large $\left.\theta_{13}\right)$;

2. Scenario n0: Normal hierarchy and $P_{H} \simeq 0$ (large $\left.\theta_{13}\right)$;

3. Scenario a1: Any hierarchy and $P_{H} \simeq 1$ (small $\left.\theta_{13}\right)$.

In the case of $P_{H} \simeq 1$ the inverted and normal hierarchy are identical. In the above scenarios large $\theta_{13}$ means a values corresponding to $\sin ^{2} \theta_{13}=10^{-3}$ and small means a value corresponding to $\sin ^{2} \theta_{13}=10^{-6}$ (see figure 1).

We will analyze three different types of next-generation experiments, namely:

- Water Cherenkov (WaterC);

- Liquid Argon (LAr);

- Scintillation.

Each of these will be discussed in detail in the following subsections. The WaterC and Scintillation experiments are much more sensitive to anti-neutrinos than neutrinos, due to the dominant inverse beta decay detection. The dominant detection channel for a LAr detector on the other hand is charged current $\nu_{e}$ interactions on Argon, making it more sensitive to neutrinos.

Before discussing the details of each detector we will note some common features. First of all, obviously having sensitivity to more than one combination of neutrino fluxes will be essential to pin down the SN and neutrino parameters. For each experiment we will make contours for two scenarios: a pessimistic one, assuming that only the dominant channel can be used, and an optimistic one, where we assume several channels can be separated by the detector. This will illustrate the necessity of having sensitivities to several channels with different sensitivity to $\nu_{e} / \bar{\nu}_{e}$ and $\nu_{x}$ fluxes. In particular, a $\mathrm{NC}$ channel along with a $\mathrm{CC}$ channel will complement each other well. A true NC channel is independent of the neutrino parameters, as is easily seen, as we have

$$
\begin{aligned}
N_{\mathrm{NC}} & \propto \int\left(F_{\nu_{e}} \sigma_{N C}+F_{\bar{\nu}_{e}} \sigma_{N C}+2 F_{\nu_{x}} \sigma_{N C}+2 F_{\bar{\nu}_{x}} \sigma_{N C}\right) d E \\
& \propto \int\left(F_{\nu_{e}}^{0}+F_{\bar{\nu}_{e}}^{0}+4 F_{\nu_{x}}^{0}\right) \sigma_{N C} d E
\end{aligned}
$$

which is given purely in terms of the original fluxes. However, it should be remembered that eg. elastic scattering on electrons is a combination of NC and CC interactions for the electron 
neutrino and anti-neutrino species. Thus for this channel $\sigma_{\bar{\nu}_{e}} \neq \sigma_{\nu_{e}} \neq \sigma_{\nu_{x}}$ and the number of events have a slight variation with the oscillation parameters.

We neglect any time-dependence of the neutrino fluxes and just look at the energy spectrum, or in the case that the energy cannot be measured, the total number of events integrated over time. The time-dependence of the energy spectra can be monitored by the experiments we study. Therefore, it will be possible to get a feeling on how well the time-independence assumption works. The SN simulations suggest that a mild steady increase in the average energies as a function of time will occur. This suggest that indeed a pinched Fermi-Dirac spectrum, can still be used, but with a broader spectrum and thus a smaller value of $\eta_{i}$. Also a steady decrease in the luminosities is expected, not influencing our method.

We will also assume that all detection efficiencies are $100 \%$ above the threshold. This is a very optimistic assumption, but the efficiencies for these future experiments are presently unknown, and at least in this manner we treat all three experiments on the same footing. Also, we will neglect the energy resolution, but this is partly compensated by the use of wide bins in energy. We will use energy bins of $10 \mathrm{MeV}$, unless otherwise specified. The exact energy resolution, which is also unknown, can become important in some cases where there are degeneracies between certain parameters. However, for most of the parameter space the exact energy resolution is not very important and will not change the general result $\sharp$.

\section{A. Analysis of a Water Cherenkov Detector}

The water Cherenkov detectors have proven very successful, with the Super-K collaboration being first at announcing extremely compelling evidence of atmospheric neutrino oscillation in 1998. The Hyper-Kamiokande [37] (Hyper-K) detector is being proposed to replace the current Super-Kamiokande experiment and will have a total mass of about one megaton. Other proposed and more or less identical detectors, with the only difference being their location and the exact mass, are the (American) UNO detector [38] and the (European) MEMPHYS detector [39]. All these detectors will of course have similar sensitivities. We assume the fiducial volume of the WaterC to be 540 kton, which is the expectation for the Hyper-Kamiokande [37] detector. Earlier works on the subject can be found in [18, 19] although these references only take into account one detection channel. As we will prove, the possibility to measure neutral current and charged current on oxygen as well as elastic scattering on electrons, will greatly improve the sensitivity of a WaterC detector.

$\sharp$ The energy resolution is, however, important for the determination of Earth matter effects for supernova neutrinos from a single detector 


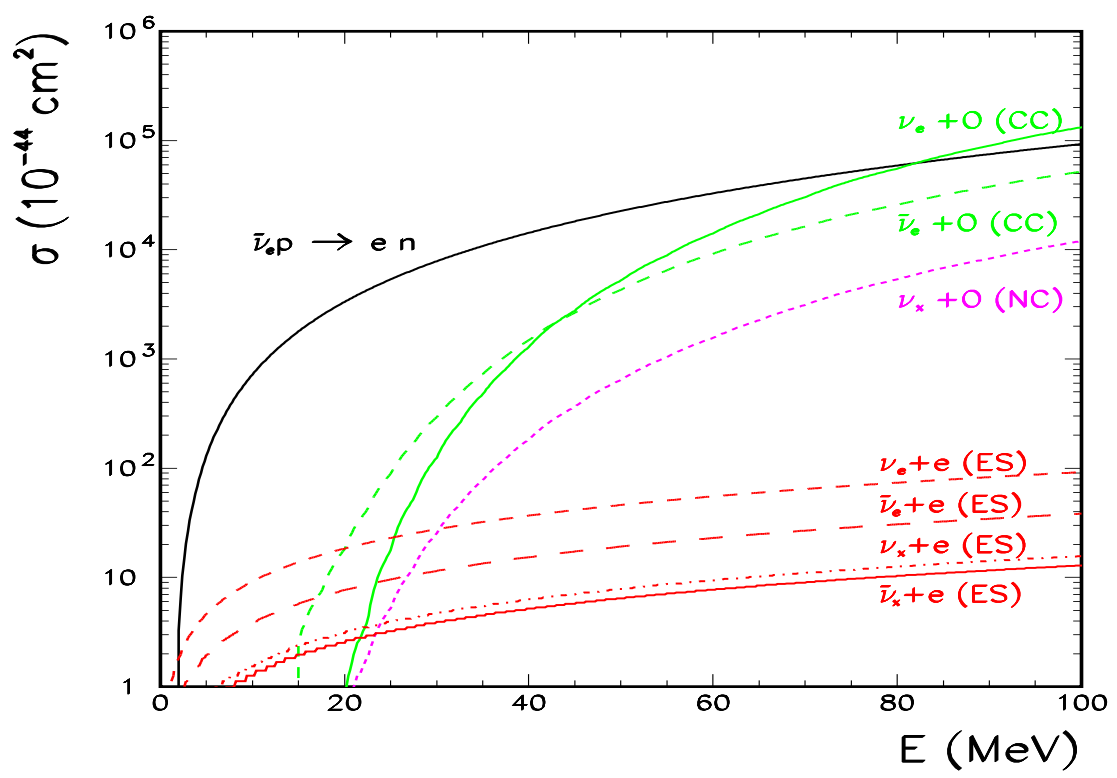

FIG. 2: The cross-section for the various channels for neutrino detection in a WaterC detector. Here ES stands for elastic scattering on electrons.

We will take into account four different channels. Two channels are CC reactions that will provide spectral information for $\nu_{e}$ and $\bar{\nu}_{e}$ fluxes. One channel is a $\mathrm{NC}$ reaction and thus sensitive to all neutrino flavors, providing information on the total neutrino flux. The ELAS channel is, as discussed earlier, also mainly an $\mathrm{NC}$ reaction, but with a small $\mathrm{CC}$ contributions. Below we list the four channels.

1. The inverse beta decay (IB) for detection of $\bar{\nu}_{e}$

$$
\bar{\nu}_{e}+p \rightarrow e^{+}+n
$$

This is by far the dominant detection channel and we will assume the threshold to be 5 $\mathrm{MeV}$. The cross-section is well-known and we use the calculation given in Ref. [40].

2. The absorption of $\nu_{e}$ and $\bar{\nu}_{e}$ on oxygen by $\mathrm{CC}$ interactions (CC-O)

$$
\stackrel{(-)}{\nu}_{e}+{ }^{16} \mathrm{O} \rightarrow e^{ \pm}+X
$$

The cross-sections are taken from Ref.[41] and the threshold for detection is about 15 $\mathrm{MeV}$.

3. The elastic scattering (ELAS) on electrons

$$
\nu_{i}+e \rightarrow \nu_{i}+e
$$


is possible for all types of neutrinos, although the cross-sections for $\nu_{e}$ and $\bar{\nu}_{e}$ are slightly higher due to the additional CC contribution. The ELAS channel is easily separated as these events are strongly forward peaked. We have set the detection threshold at $7 \mathrm{MeV}$.

4. An interesting channel for observation is the excitation of oxygen by NC interactions, followed by a decay chain with emission of a detectable mono-energetic photon, as first discussed in Ref.[42],

$$
\nu_{i}+{ }^{16} \mathrm{O} \rightarrow \nu_{i}+\gamma+X
$$

Hyper-K can detect photons with an energy greater than about $5 \mathrm{MeV}$. The excited ${ }^{16} \mathrm{O}$ atom decay to either ${ }^{15} \mathrm{O}^{*}$ or ${ }^{15} \mathrm{~N}^{*}$, which then emits a photon with the energy in the range 5-10 MeV. As the photon has a well defined energy, these events can be easily separated from the other detection channels. We will refer to this channel as NC-O, even though it should be remember that it only includes those partial decay chains which give rise to a detectable mono-energetic photon. This channel differs from the others as the energy spectrum cannot be measured. Henceforth we only use the total number of events.

We will for the main parts of this article assume that the four channels can be separated, and make remarks on the case where only the inverse beta decay channel is detectable. The NC-O and ELAS channels should be easily separated from the others. As discussed in Ref.[31], the IB events should be slightly forward peaked, whereas the CC-O events should be slightly backward peaked and this gives an opportunity to distinguish these events. Moreover, by addition of small amounts of gadolinium [43] in the WaterC detector, the capture of neutrons is possible and this would assure the separation of the IB and CC-O channels. This is also the reason that we have put the detection threshold for the IB channel as low as $5 \mathrm{MeV}$. Presumably even a lower threshold can be achieved with a gadolinium enriched WaterC detector. For the IB, CC-O and ELAS channels we calculate the energy spectrum and we use 10 bins with a width of $10 \mathrm{MeV}$ and the first bin starting at $5 \mathrm{MeV}$. In Figure 2 we show the cross-sections involved.

In Table II we show the total number of events we calculate for the SN parameters at point 1. The dominant channel for Hyper- $\mathrm{K}$ is the inverse beta decay channel, which is only sensitive to the $\bar{\nu}_{e}$ flux arriving at the Earth. However, the NC-O, CC-O and ELAS channel is very important for having sensitivity to other combination of fluxes. Moreover, the ELAS channel gives the opportunity to determine the direction to the supernova and thus an early warning to astronomers will be possible. From Table II is expected that if the true neutrino parameters are consistent with the inverted hierarchy and large $\theta_{13}$ then Hyper-K will be able to determine this scenario with a very high confidence level. But, even the sub-dominant channel allows for a determination of the normal hierarchy if the angle $\theta_{13}$ is sufficiently large. In section IV we 


\begin{tabular}{|c||r|r|r|}
\hline $\begin{array}{c}\text { Expected Number of Events in a } 540 \text { kton water Cherenkov detector } \\
\left\langle E_{e}\right\rangle=12 \mathrm{MeV},\left\langle E_{\bar{e}}\right\rangle=15 \mathrm{MeV},\left\langle E_{x}\right\rangle=18 \mathrm{MeV}, L_{x}=1.5 L_{e}\end{array}$ \\
$\begin{array}{c}\text { Reaction } \\
\text { Inverse beta decay }\end{array}$ & $1.4 \times 10^{5}$ & $2.1 \times 10^{5}$ & $\begin{array}{c}\text { Normal hierarchy } \\
\text { Large } \theta_{13}\end{array}$ \\
\hline \hline${ }^{(-)}$CC on oxygen & $7.7 \times 10^{3}$ & $10.7 \times 10^{3}$ & $1.4 \times 10^{5}$ \\
\hline$\nu_{x}+e \rightarrow \nu_{x}+e$ & $8.4 \times 10^{3}$ & $8.7 \times 10^{3}$ & $9.5 \times 10^{3}$ \\
\hline NC on oxygen & $3.5 \times 10^{3}$ & $3.5 \times 10^{3}$ & $8.8 \times 10^{3}$ \\
\hline \hline TOTAL & $1.63 \times 10^{5}$ & $2.34 \times 10^{5}$ & $3.5 \times 10^{3}$ \\
\hline \hline
\end{tabular}

TABLE II: Expected number of neutrino events in a 540 kton water Cherenkov detector for the SN parameters at point 1 and each of the cases a1, i0 and n0 for the neutrino parameters.

will comment on how this dependent on the SN parameters (luminosities and average energies), by using our reference points.

\section{B. Analysis of a LAr Experiment}

Next, we will look at the possibility to determine the supernova and neutrino parameters at a future Liquid Argon experiment. The are various LAr experiments proposed. The Icarus detector at CNGS is expected to have a 3 kton final version ${ }^{\dagger}$ and already a 300 ton detector is running. Moreover, the LANNDD [44], the GLACIER [45] and the Flare [46] detectors are being discussed as possible future detectors in the 100 kton size. Earlier works on the subject can be found in [20, 28, 47, 48].

We will take into account the following channels:

1. Detection of $\nu_{e}$ through CC-interaction:

$$
\nu_{e}+{ }^{40} \mathrm{Ar} \rightarrow e^{-}+A^{\prime}+\mathrm{nN} \quad\left(E_{\mathrm{thr}}=1.5 \mathrm{MeV}\right)
$$

where $\mathrm{nN}$ represent emitted nucleons or other debris (like $\alpha$-particles etc.) and $A^{\prime}$ is the leftover nucleus. The CC cross-section on Argon are taken from Ref. [48, 49].

2. Detection of $\bar{\nu}_{e}$ through CC-interaction:

$$
\bar{\nu}_{e}+{ }^{40} \mathrm{Ar} \rightarrow e^{+}+A^{\prime}+\mathrm{nN}
$$

having a threshold about $7.5 \mathrm{MeV}$.

\footnotetext{
†Unfortunately, ICARUS might be interrupted due to cancellation of its funding.
} 


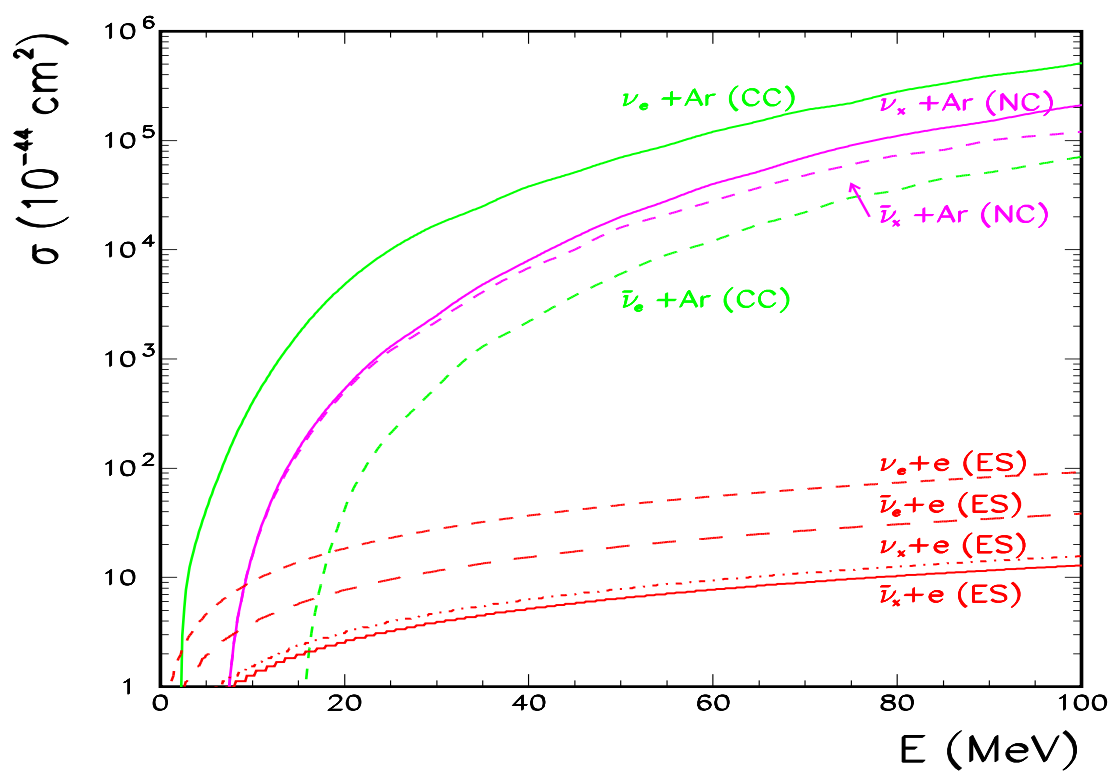

FIG. 3: The cross-section for the various channels of neutrino detection in LAr.

3. The elastic scattering (ELAS) on electrons

$$
\nu_{x}+e \rightarrow \nu_{x}+e
$$

is possible for all flavors of neutrinos. Again we have taken the threshold to be $7 \mathrm{MeV}$.

4. The scattering on Argon of any type of neutrino through NC-interaction:

$$
\nu_{i}+{ }^{40} \mathrm{Ar} \rightarrow \nu_{i}+A^{\prime}+\mathrm{nN} .
$$

This channel has no sensitivity to the energy. The NC cross-section on Argon are taken from Ref. [48, 49].

As in Ref.[20] it is assumed that one can separate all four channels. However, we will also make contours for the 'worse case' scenario were only detection in the $\nu_{e} \mathrm{CC}$ channel is available. For the $\nu_{e}$ and $\bar{\nu}_{e} \mathrm{CC}$ reactions and the $\nu_{i} \mathrm{NC}$ reactions, the energy and time-delay of the photons emitted from the de-excitation of respectively $\mathrm{K}, \mathrm{Cl}$ and $\mathrm{Ar}$ can be used to classify the type of event. For the ELAS events no photons will be present. The cross-sections are shown in Figure 3, and it should be noted that we do not take into account in our calculations their uncertainties. At the moment there are no experimental confirmation of the theoretically calculated cross-sections. But hopefully, in the case that a large scale LAr detector will be realized, the cross-sections will already have been experimentally measured (eg. by ICARUS). For the channels with measurable energy spectra we again use 10 energy bins of $10 \mathrm{MeV}$ each, the first bin starting at $5 \mathrm{MeV}$. 


\begin{tabular}{|c||r|r|r|}
\hline \multicolumn{3}{|c|}{$\begin{array}{c}\text { Expected Number of Events in a } 100 \text { kton LAr detector } \\
\left\langle E_{e}\right\rangle=12 \mathrm{MeV},\left\langle E_{\bar{e}}\right\rangle=15 \mathrm{MeV},\left\langle E_{x}\right\rangle=18 \mathrm{MeV}, L_{x}=1.5 L_{e}\end{array}$} \\
\hline Reaction & $\begin{array}{c}\text { Any hierarchy } \\
\text { Small } \theta_{13}\end{array}$ & $\begin{array}{c}\text { Inverted hierarchy } \\
\text { Large } \theta_{13}\end{array}$ & $\begin{array}{c}\text { Normal hierarchy } \\
\text { Large } \theta_{13}\end{array}$ \\
\hline \hline$\nu_{e}$ CC on Argon & $1.4 \times 10^{4}$ & $1.4 \times 10^{4}$ & $1.7 \times 10^{4}$ \\
\hline $\bar{\nu}_{e}$ CC on Argon & $4.2 \times 10^{2}$ & $7.9 \times 10^{2}$ & $4.2 \times 10^{2}$ \\
\hline ELAS & $1.2 \times 10^{3}$ & $1.3 \times 10^{3}$ & $1.3 \times 10^{2}$ \\
\hline NC on Argon & $1.3 \times 10^{4}$ & $1.3 \times 10^{4}$ & $1.3 \times 10^{2}$ \\
\hline \hline TOTAL & $2.80 \times 10^{4}$ & $2.84 \times 10^{4}$ & $3.16 \times 10^{4}$ \\
\hline
\end{tabular}

TABLE III: Expected number of neutrino events at a 100 kton LAr detector for the SN parameters at point 1 and each of the cases a1, i0 and $n 0$ for the neutrino parameters.

In Table III we show the total number of events for the SN parameters at point 1 for each detection channel. The dominant channel is detection of $\nu_{e}$ by the charged current interaction on Argon. Also the NC and ELAS channels have a fairly large number of events, whereas the sensitivity to the $\bar{\nu}_{e}$ flux is rather weak.

\section{Analysis of a Scintillation Detector}

Finally, we will examine the proposal for the 50 kton Low Energy Neutrino Astronomy (LENA) [50, 51] liquid scintillation detector. We assume that LENA will be filled with pure PXE $\left(\mathrm{C}_{16} \mathrm{H}_{18}\right)$. If another oil will be used the carbon to proton ratio may change and thus the results will change slightly. A discussion on SN neutrinos and scintillator detectors can be found in [51, 52], although without an explicit calculation of the accuracy of the determination of the parameters.

We will exploit six $\nu$ detection channels in LENA (three are CC reactions, two are NC reactions and the last is the ELAS channel), these are listed below:

1. The inverse beta decay for detection of $\bar{\nu}_{e}$

$$
\bar{\nu}_{e}+p \rightarrow e^{+}+n
$$

The threshold $\bar{\nu}_{e}$ energy for this reaction is $1.8 \mathrm{MeV}$. Again we take the IB cross-section from [40].

2. The CC capture of $\bar{\nu}_{e}$ on ${ }^{12} \mathrm{C}$

$$
\begin{aligned}
& \bar{\nu}_{e}+{ }^{12} \mathrm{C} \rightarrow{ }^{12} \mathrm{~B}+e^{+} \\
& { }^{12} \mathrm{~B} \rightarrow{ }^{12} \mathrm{C}+e^{-}+\bar{\nu}_{e}
\end{aligned}
$$




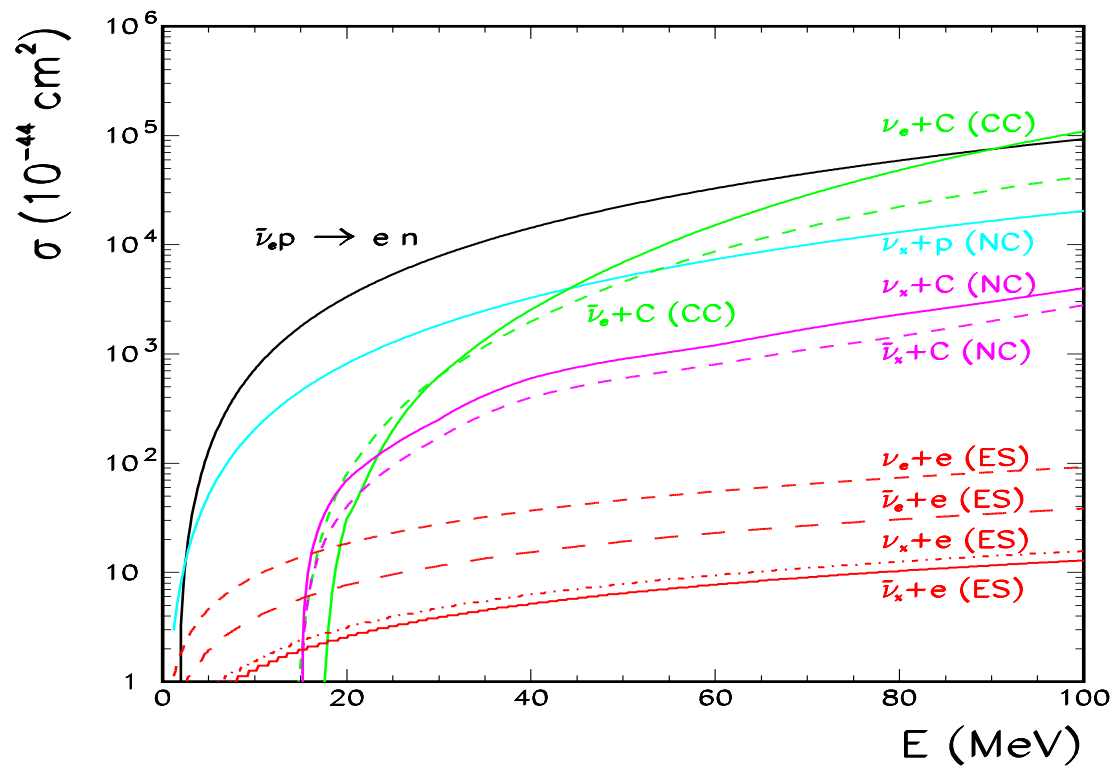

FIG. 4: The cross-section for the various channels of neutrino detection in LENA.

The threshold $\bar{\nu}_{e}$ energy for the capture on ${ }^{12} \mathrm{C}$ is $14.39 \mathrm{MeV}$.

3. The CC capture of $\nu_{e}$ on ${ }^{12} \mathrm{C}$

$$
\begin{aligned}
& \nu_{e}+{ }^{12} \mathrm{C} \rightarrow{ }^{12} \mathrm{~N}+e^{-} \\
& { }^{12} \mathrm{~N} \rightarrow{ }^{12} \mathrm{C}+e^{+}+\nu_{e}
\end{aligned}
$$

The threshold neutrino energy for capture on ${ }^{12} \mathrm{C}$ is $17.34 \mathrm{MeV}$.

4. Elastic scattering on protons

$$
\nu_{i}+p \rightarrow \nu_{i}+p
$$

This process might in some areas of parameters space even give a larger number of events than the IB process, due to the factor of six, originating from the number of neutrino and anti-neutrino species. The cross-sections is taken from [53], where we have used the approximation of equal cross-section for neutrinos and anti-neutrinos. We have implemented a neutrino threshold energy of $25 \mathrm{MeV}$, corresponding roughly to a cut of $0.2 \mathrm{MeV}$ in electron equivalent energy.

5. $\mathrm{NC}$ scattering on ${ }^{12} \mathrm{C}$ :

$$
\begin{aligned}
& \nu_{i}\left(\overline{\nu_{i}}\right)+{ }^{12} \mathrm{C} \rightarrow{ }^{12} \mathrm{C}^{*}+\nu_{i}^{\prime}\left(\overline{\nu_{i}^{\prime}}\right), \\
& { }^{12} \mathrm{C}^{*} \rightarrow{ }^{12} \mathrm{C}+\gamma(15.11 \mathrm{MeV})
\end{aligned}
$$




\begin{tabular}{|c||r|r|c|}
\hline $\left.\begin{array}{c}\text { Expected Number of Events in a } 50 \text { kton Scintillation Detector } \\
\left\langle E_{e}\right\rangle=12 \mathrm{MeV},\end{array} E_{\bar{e}}\right\rangle=15 \mathrm{MeV},\left\langle E_{x}\right\rangle=18 \mathrm{MeV}, L_{x}=1.5 L_{e}$ \\
\hline & $\begin{array}{c}\text { Any hierarchy } \\
\text { Reaction }\end{array}$ & $\begin{array}{c}\text { Inverted hierarchy } \\
\text { Small } \theta_{13}\end{array}$ & $\begin{array}{c}\text { Normal hierarchy } \\
\text { Large } \theta_{13}\end{array}$ \\
\hline Inverse beta decay & $1.0 \times 10^{4}$ & $1.5 \times 10^{4}$ & $1.0 \times 10^{4}$ \\
\hline $\bar{\nu}_{e}$ CC on carbon & $6.0 \times 10^{2}$ & $1.1 \times 10^{3}$ & $6.0 \times 10^{2}$ \\
\hline$\nu_{e}$ CC on carbon & $1.0 \times 10^{3}$ & $1.0 \times 10^{3}$ & $1.4 \times 10^{3}$ \\
\hline$\nu_{i}$ NC on proton & $9.9 \times 10^{3}$ & $9.9 \times 10^{3}$ & $9.9 \times 10^{3}$ \\
\hline$\nu_{i}+e \rightarrow \nu_{i}+e$ & $7.9 \times 10^{2}$ & $8.2 \times 10^{2}$ & $8.2 \times 10^{2}$ \\
\hline$\nu_{i}$ NC on carbon & $1.4 \times 10^{3}$ & $1.4 \times 10^{3}$ & $1.4 \times 10^{3}$ \\
\hline \hline TOTAL & $2.39 \times 10^{4}$ & $2.93 \times 10^{4}$ & $2.43 \times 10^{4}$ \\
\hline
\end{tabular}

TABLE IV: Expected number of neutrino events in a 50 kton scintillation detector for the SN parameters at point 1 and each of the cases a1, i0 and n0 for the neutrino parameters.

The emission of a mono-energetic photon, makes this channel easily separated from the others. This cross-section is taken from [54]. Since the emitted photon carries no information about the neutrino energy, this is the only channel for which LENA will have no energy information. Therefore, we will only use the total number of events from this process.

6. ELAS on electrons

$$
\nu_{i}+e \rightarrow \nu_{i}+e
$$

which has been discussed in earlier sections.

Here again we take two approaches. The conservative one, where we consider only the inverse beta decay channel and the optimistic one, where we assume that all channels can be distinguished from each other. In principle, one can hope that, due to the distinctive signatures of the above discussed channels they can be separated. The most doubtful discrimination is between the $\nu_{e}$ and $\bar{\nu}_{e}$ CC reactions on carbon. It might be possible to separate these by using the delayed coincidence of the $\beta^{+} / \beta^{-}$decays with the primarily produced electron/positron and the knowledge of the average lifetimes of the produced unstable nuclei. For the channels with sensitivity to the neutrino energy spectra (all except the NC scattering on carbon) we again use 10 energy bins of $10 \mathrm{MeV}$ each, the first bin starting at $5 \mathrm{MeV}$. Moreover, due to the very fine energy resolution expected for LENA, with a threshold of order $200 \mathrm{keV}$, we also include an extra low-energy bin, with the events originating from neutrino energies below 5 $\mathrm{MeV}$, for the IB and ELAS channels.

The dominant channels for LENA are the inverse beta decay channel (for $\left.\bar{\nu}_{e}\right)$ and the $\nu_{i} \mathrm{NC}$ scattering on protons. In Table [V] we show the total number of events for the SN parameters at 
point 1 for each observable neutrino channel in LENA. The detector will have a large sensitivity to both $\bar{\nu}_{e}$ and the total neutrino flux and even a reasonable sensitivity to the $\nu_{e}$ flux.

\section{RESULTS}

In this section we will study two scenarios for the detection capability for each detector: the case where only the main CC channel is available and the case where all channels discussed in section III are available and moreover separable. As detecting only the main CC channel is a rather pessimistic scenario, we include in appendix A a discussion of the detector performance in a few other cases with a more realistic, but non-optimal, detector-setup. The main reasons for studying the main CC channel only case is to compare to other studies (Refs. [18, 19]) and also to illustrate the importance of having both $\mathrm{NC}$ and $\mathrm{CC}$ channels. Furthermore, this is the absolute worst case scenario and with the two contours considered in this section we give the span of possible allowed regions.

In Figs. 5, 6 and 7 we show the $3 \sigma$ CL allowed regions obtained for a WaterC detector in the three limiting cases a1, i0 and n0, discussed in Sec. III. The corresponding regions for the LAr and the Scintillation detectors are shown in respectively Figs. 8, 9] and 10, and Figs. 11, 12 and 13.

In the case a1 the hierarchy cannot be determined, as both hierarchies produce the same neutrino fluxes. All three experiments present a good sensitivity to $\theta_{13}$ as can be seen from Figs. 5, 8 and 11 and will provide an upper limit on $\sin ^{2} \theta_{13}$ of about $1-2 \times 10^{-5}$, if there are several channels available. In the case that only the main channel is available (IB or $\nu_{e} \mathrm{CC}$ ) a degeneracy in $\theta_{13}$ and the hierarchy occurs and $\theta_{13}$ cannot be determined (unless the hierarchy has already been established by another experiment). This independence of $\theta_{13}$ for the case of normal hierarchy and the WaterC and Scintillator detectors, is evident from Fig.1 as the IB channel is only sensitive to $P_{\bar{e} \bar{e}}$, which is almost constant as a function of $\theta_{13}$. Similarly, the LAr detector is not sensitive to $\theta_{13}$ in the case that only the $\nu_{e} \mathrm{CC}$ channel is available and the hierarchy is inverted. The anti-neutrino flux arriving at Earth for the case a1 (and n0) consist of roughly $70 \%$ with a temperature of $15 \mathrm{MeV}$ and $30 \%$ with a temperature of $18 \mathrm{MeV}$ (for point 1). For the Scintillator detector the statistics for the only IB case is such that a pure flux with temperature of about $16.5 \mathrm{MeV}$ can also fit this data. Therefore, the inverted hierarchy is allowed even for large values of $\theta_{13}$. However, for a small interval around $\sin ^{2} \theta_{13} \simeq 10^{-4}$ and low values of $\left\langle E_{\bar{e}}\right\rangle$ it is not possible to fit the energy spectrum, giving rise to islands in the contours as seen on Fig 11 (the exactly same contours are found in Fig 13). If $\sin ^{2} \theta_{13}$ turns out to be in the region where $P_{H} \simeq 1$, then there is no possibility that it can be determined by any of the laboratory experiments currently proposed [55, 56, 57, 58]. In this case SN information 

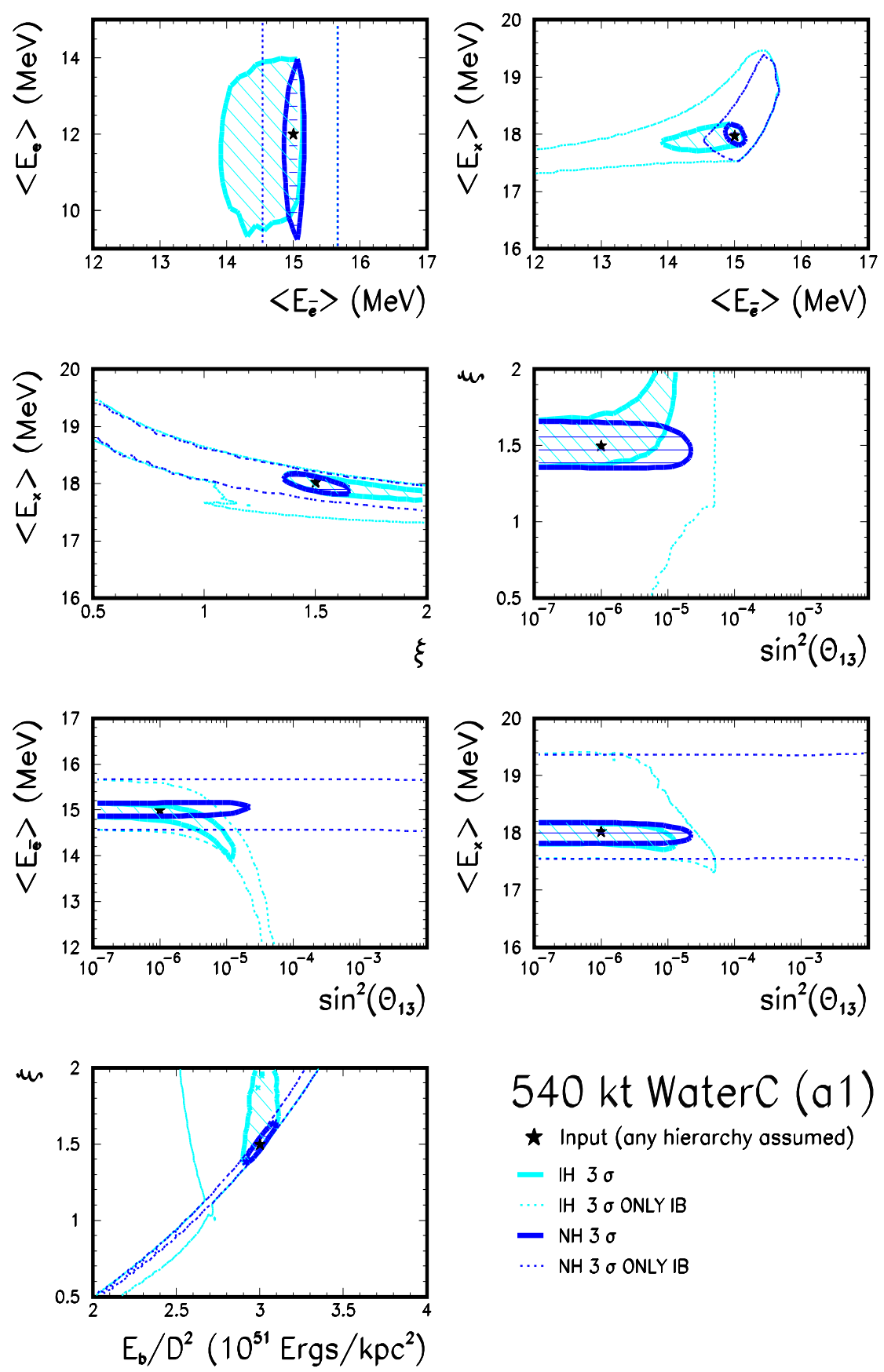

$\star$ Input (any hierarchy assumed)

$-\mathrm{H} 3 \sigma$

… IH $3 \sigma$ ONLY IB

- $\mathrm{NH} 3 \sigma$

.... NH $3 \sigma$ ONLY IB

FIG. 5: Sensitivity of a 540 kton WaterC detector assuming true SN parameters as in point 1 of Table I, for any hierarchy and $\sin ^{2}\left(\theta_{13}\right)=10^{-6}\left(P_{H} \simeq 1\right)$. We show $3 \sigma$ CL contours (2 dof) using: all 4 channels (IB + CC-O + ELAS + NC-O) and normal hierarchy (NH) marked by the dark (blue) horizontally hatched area; only the IB channel and normal hierarchy marked by the dark (blue) dashed line; all 4 channels and inverted hierarchy (IH) marked by the light (cyan) diagonally hatched area; only the IB channel and inverted hierarchy marked by the light (cyan) dashed line. 

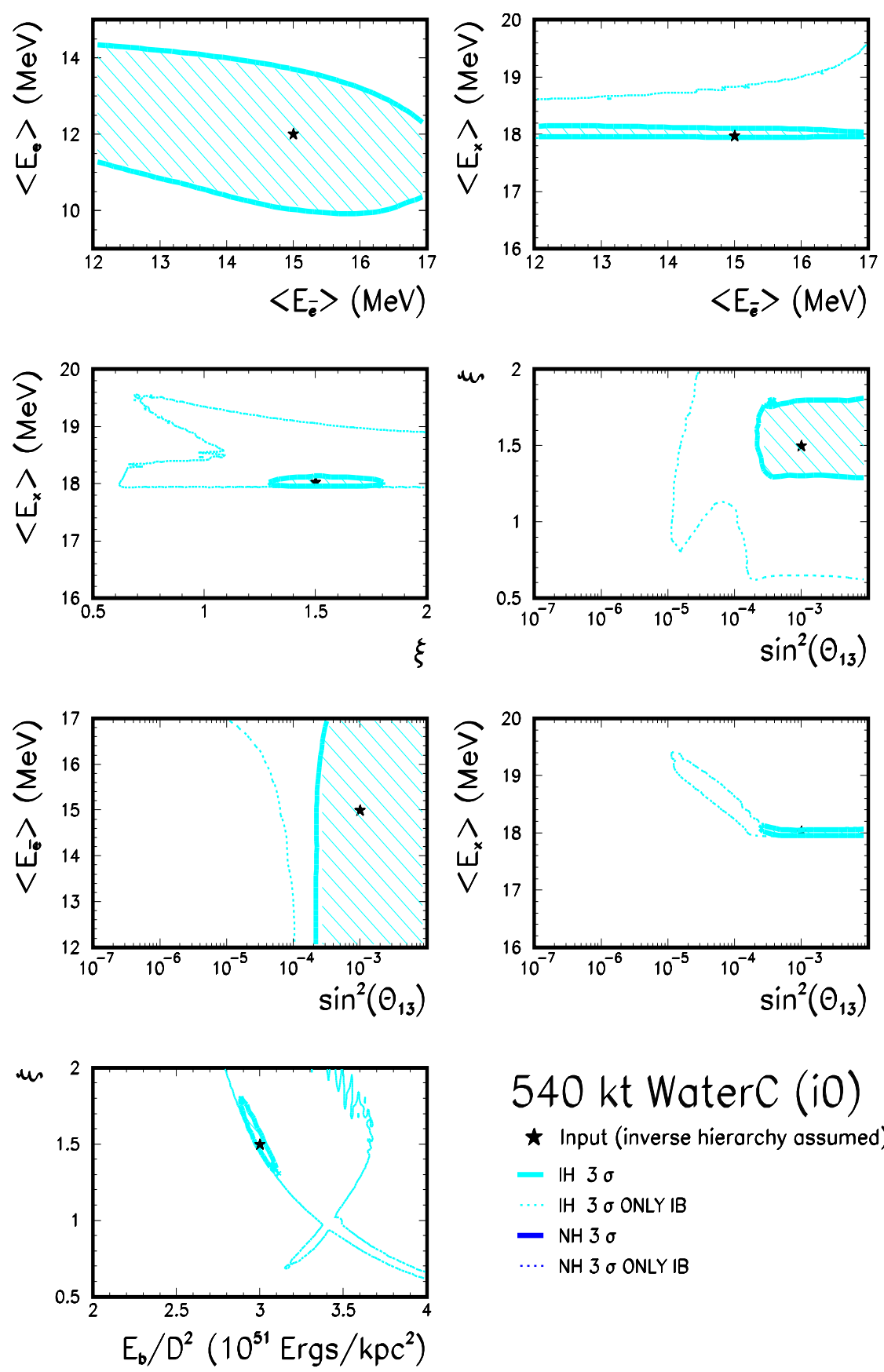

\section{$540 \mathrm{kt}$ WaterC (i0)}

$\star$ Input (inverse hierarchy assumed)

$-1 \mathrm{H} 3 \sigma$

$\cdots \quad$ IH $3 \sigma$ ONLY IB

- $\mathrm{NH} 3 \sigma$

… NH $3 \sigma$ ONLY IB

FIG. 6: Same as Fig. 5 but for the inverted hierarchy and $\sin ^{2}\left(\theta_{13}\right)=10^{-3}\left(P_{H} \simeq 0\right)$. The normal hierarchy is ruled out by more than $5 \sigma$, having a global $\chi_{\min }^{2} \simeq 900$ in the case of all four channels being present. 

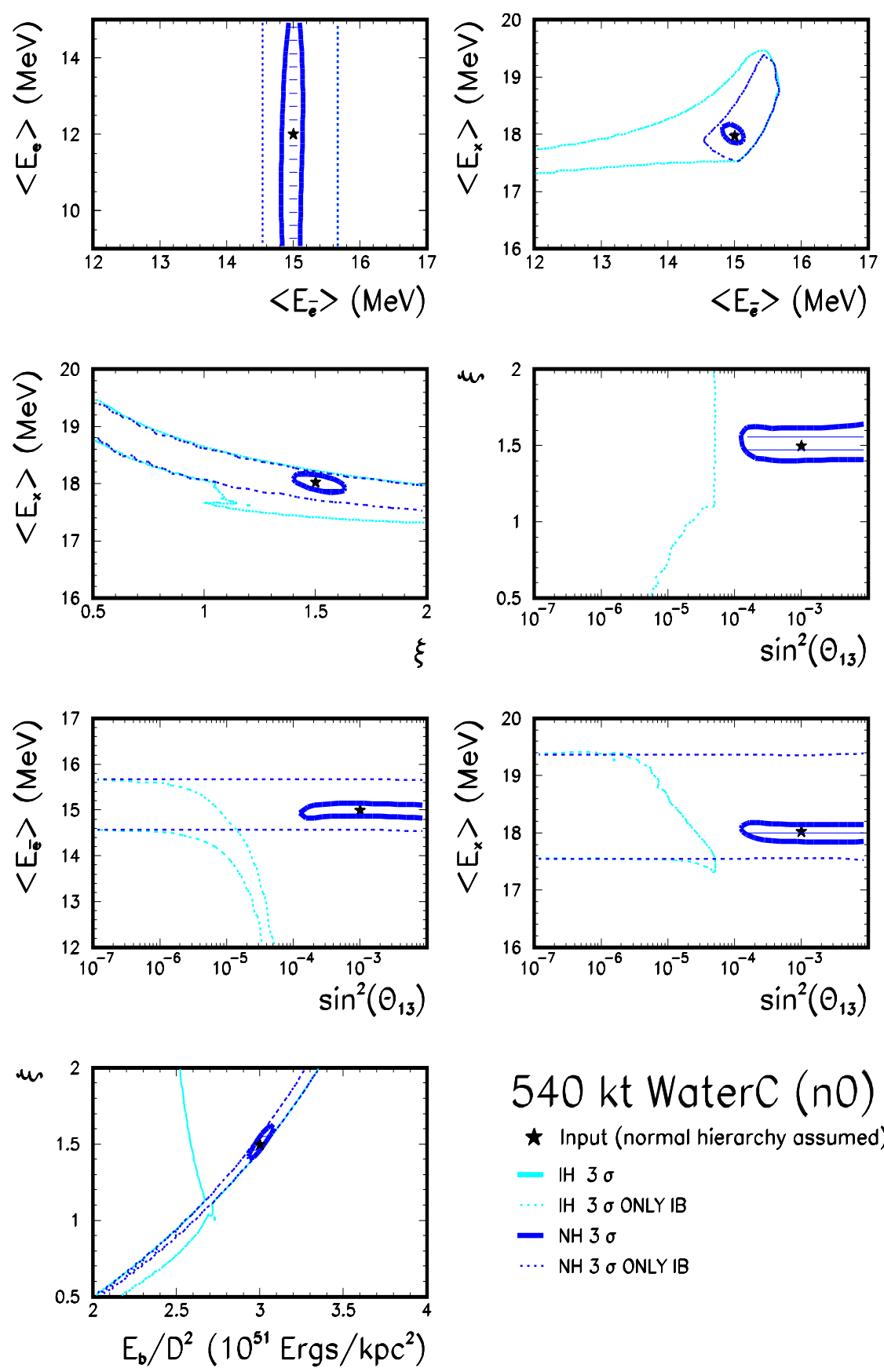

\section{$540 \mathrm{kt}$ WaterC (nO)}

$\star$ Input (normal hierarchy assumed)

$-\mathbb{H} 3 \sigma$

… IH $3 \sigma$ ONLY IB

$-\mathrm{NH} 3 \sigma$

... NH $3 \sigma$ ONLYIB

FIG. 7: Same as Fig. 5 but for the normal hierarchy and $\sin ^{2}\left(\theta_{13}\right)=10^{-3}\left(P_{H} \simeq 0\right)$. The inverted hierarchy is ruled out by more than $5 \sigma$, having a global $\chi_{\min }^{2}=160$ in the case of all four channels being present. 

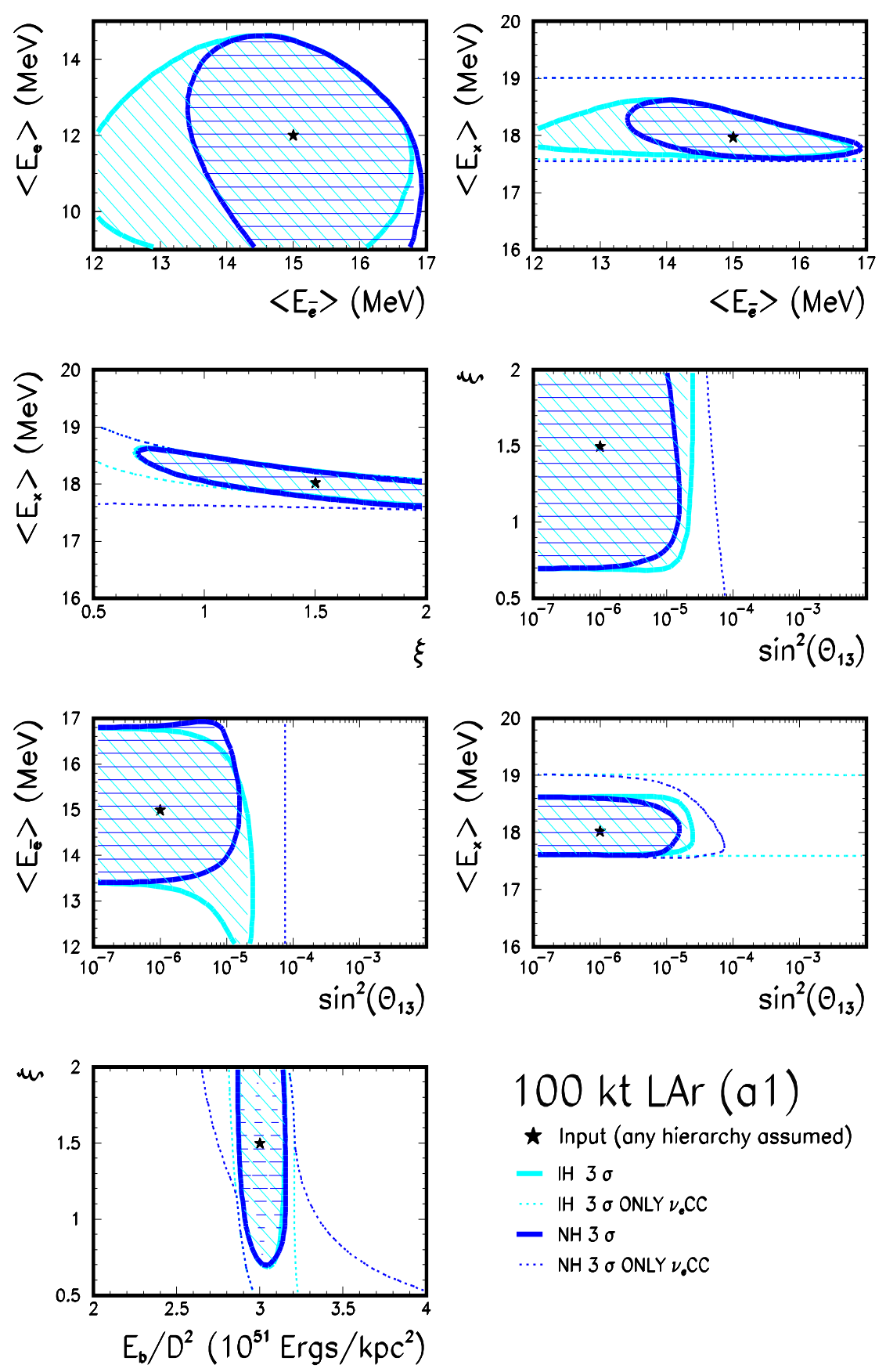

\section{$100 \mathrm{kt} \operatorname{LAr}(\mathrm{a} 1)$}

$\star$ Input (any hierarchy assumed)

$-\mathrm{H} 3 \sigma$

… IH $3 \sigma$ ONLY $\nu_{e} C C$

- $\mathrm{NH} 3 \sigma$

-... $\mathrm{NH} 3 \sigma$ ONLY $\nu_{\mathrm{e}} \mathrm{CC}$

FIG. 8: Sensitivity of a 100 kton LAr detector assuming true SN parameters as in point 1 of Table I. for any hierarchy and $\sin ^{2}\left(\theta_{13}\right)=10^{-6}\left(P_{H} \simeq 1\right)$. We show $3 \sigma$ CL contours (2 dof) using: all 4 channels $\left(\nu_{e} \mathrm{CC}+\bar{\nu}_{e} \mathrm{CC}+\mathrm{ELAS}+\mathrm{NC}\right)$ and normal hierarchy marked by the dark (blue) horizontally hatched area; only the $\nu_{e} \mathrm{CC}$ channel and normal hierarchy marked by the dark (blue) dashed line; all 4 channels and inverted hierarchy marked by the light (cyan) diagonally hatched area; only the $\nu_{e} \mathrm{CC}$ channel and inverted hierarchy marked by the light (cyan) dashed line. 

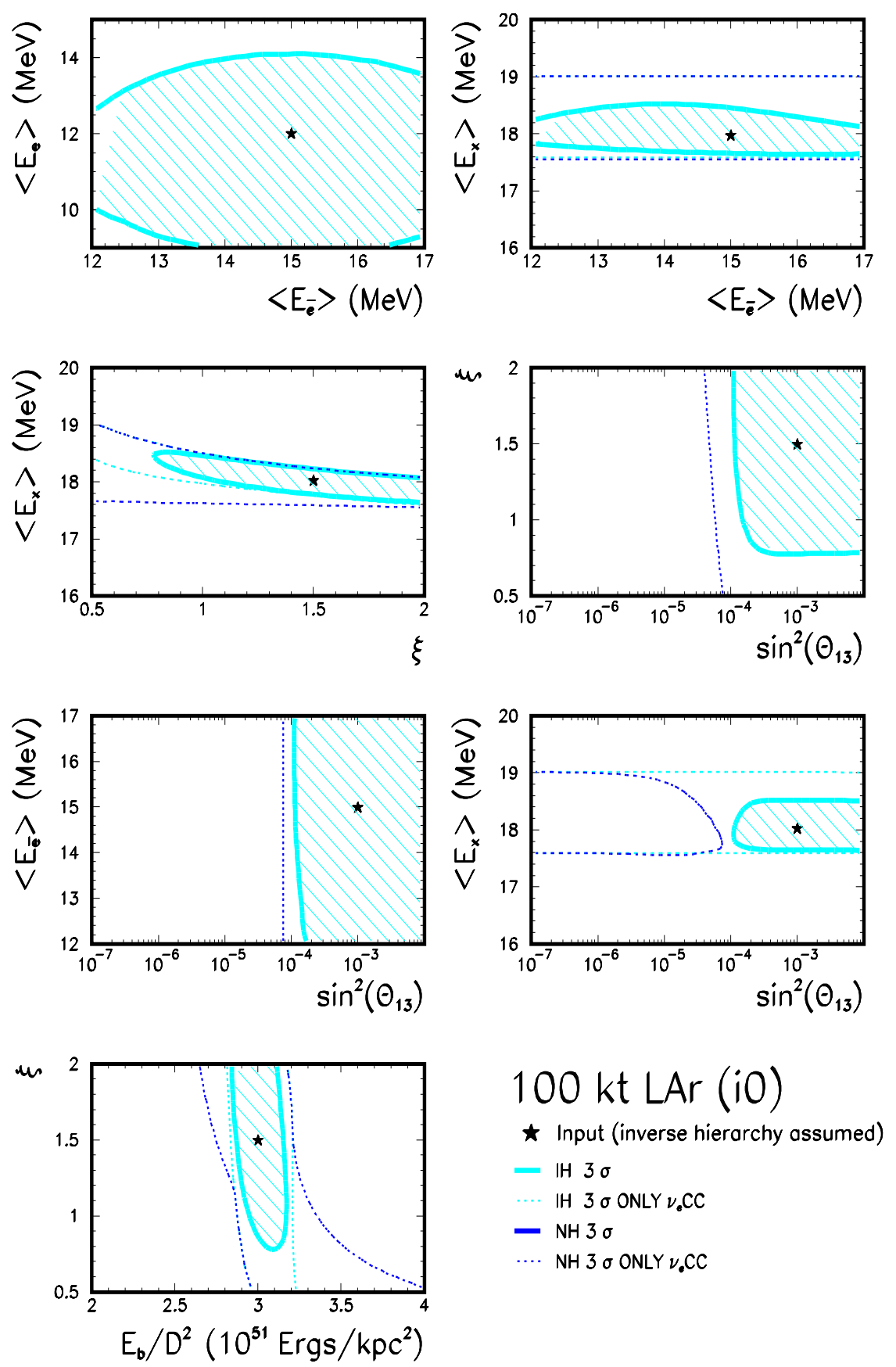

FIG. 9: Same as Fig. 8 but for the inverted hierarchy and $\sin ^{2}\left(\theta_{13}\right)=10^{-3}\left(P_{H} \simeq 0\right)$. The normal hierarchy is ruled out by more than $4 \sigma$, having a global $\chi_{\min }^{2}=20$ in the case of all four channels being present. 

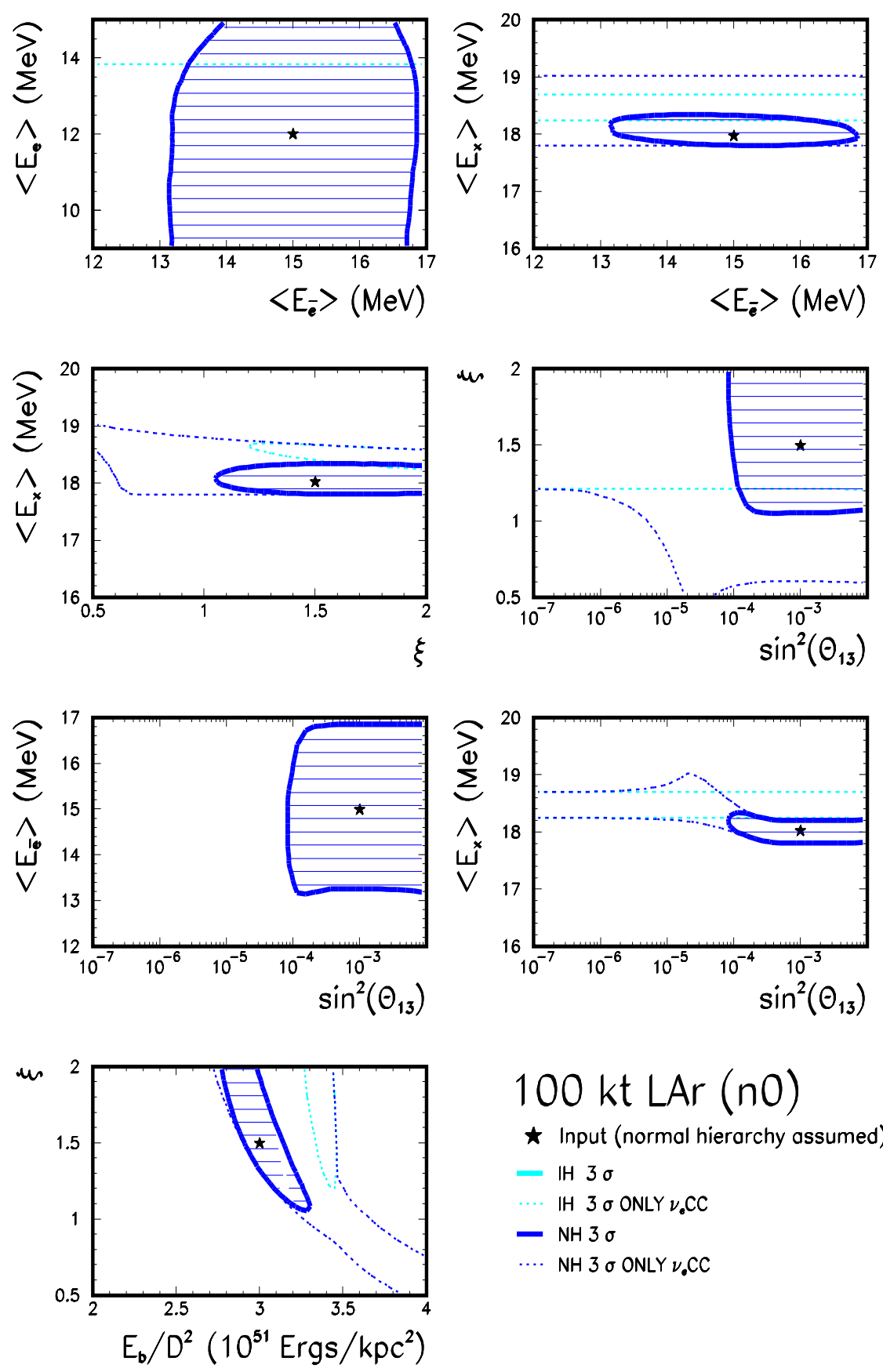

$\star$ Input (normal hierarchy assumed)

$-\mathbb{H} 3 \sigma$

… IH $3 \sigma$ ONLY $\nu_{e} C C$

$-\mathrm{NH} 3 \sigma$

-... $\mathrm{NH} 3 \sigma$ ONLY $\nu_{\mathrm{e}} \mathrm{CC}$

FIG. 10: Same as Fig. 8 but for the normal hierarchy and $\sin ^{2}\left(\theta_{13}\right)=10^{-3}\left(P_{H} \simeq 0\right)$. The inverted hierarchy is ruled out by more than $5 \sigma$, having a global $\chi_{\min }^{2}=280$ in the case of all four channels being present. 

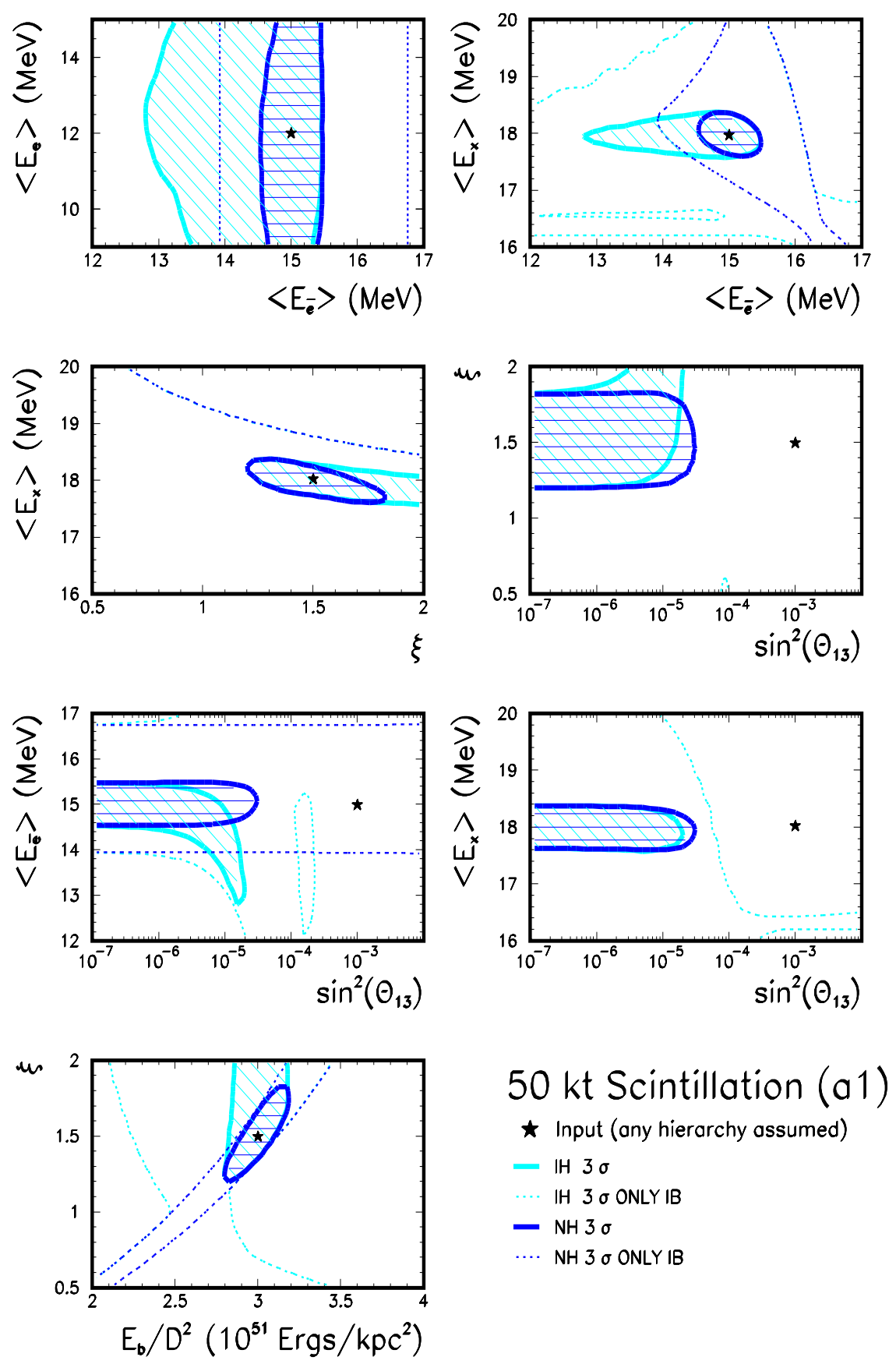

$\star$ Input (any hierarchy assumed)

$-\mathrm{H} 3 \sigma$

… IH $3 \sigma$ ONLY IB

- $\mathrm{NH} 3 \sigma$

.... NH $3 \sigma$ ONLYIB

FIG. 11: Sensitivity of a 50 kton Scintillation detector assuming true SN parameters as in point 1 of Table I, for any hierarchy and $\sin ^{2}\left(\theta_{13}\right)=10^{-6}\left(P_{H} \simeq 1\right)$. We show $3 \sigma$ CL contours $(2$ dof $)$ using: all 6 channels (IB $+\nu_{e} \mathrm{CC}+\bar{\nu}_{e} \mathrm{CC}+\nu-p+\mathrm{NC}+$ ELAS) and normal hierarchy marked by the dark (blue) horizontally hatched area; only the IB channel and normal hierarchy marked by the dark (blue) dashed line; all 4 channels and inverted hierarchy marked by the light diagonally (cyan) hatched area; only the IB channel and inverted hierarchy marked by the light (cyan) dashed line. 

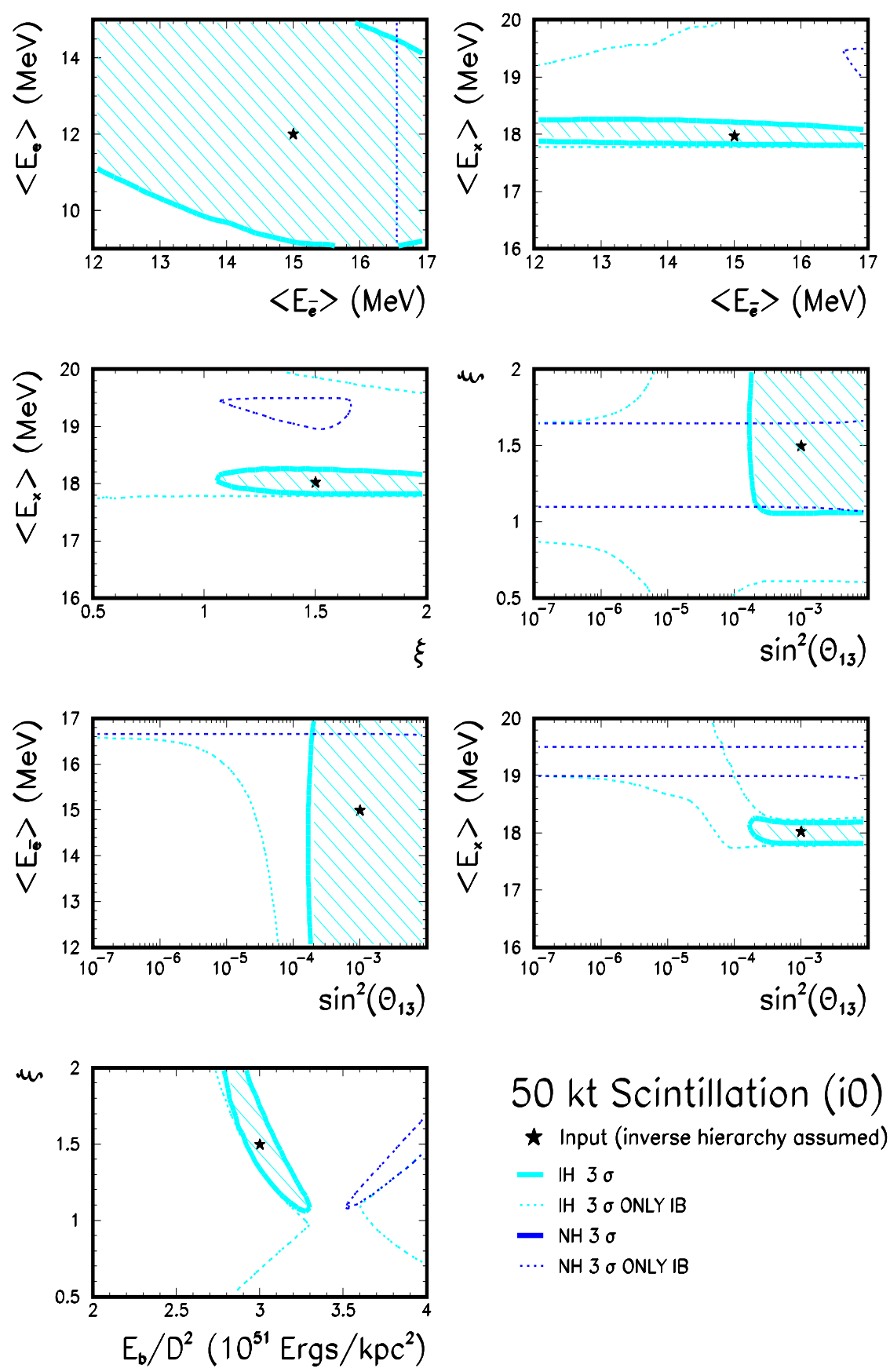

$\star$ Input (inverse hierarchy assumed)

$-\mathrm{H} 3 \sigma$

… IH $3 \sigma$ ONLY IB

- $\mathrm{NH} 3 \sigma$

.... NH $3 \sigma$ ONLYIB

FIG. 12: Same as Fig. 11 but for the inverted hierarchy and $\sin ^{2}\left(\theta_{13}\right)=10^{-3}\left(P_{H} \simeq 0\right)$. The normal hierarchy is ruled out by more than $5 \sigma$, having a global $\chi_{\min }^{2}=120$ in the case of all six channels being present. 

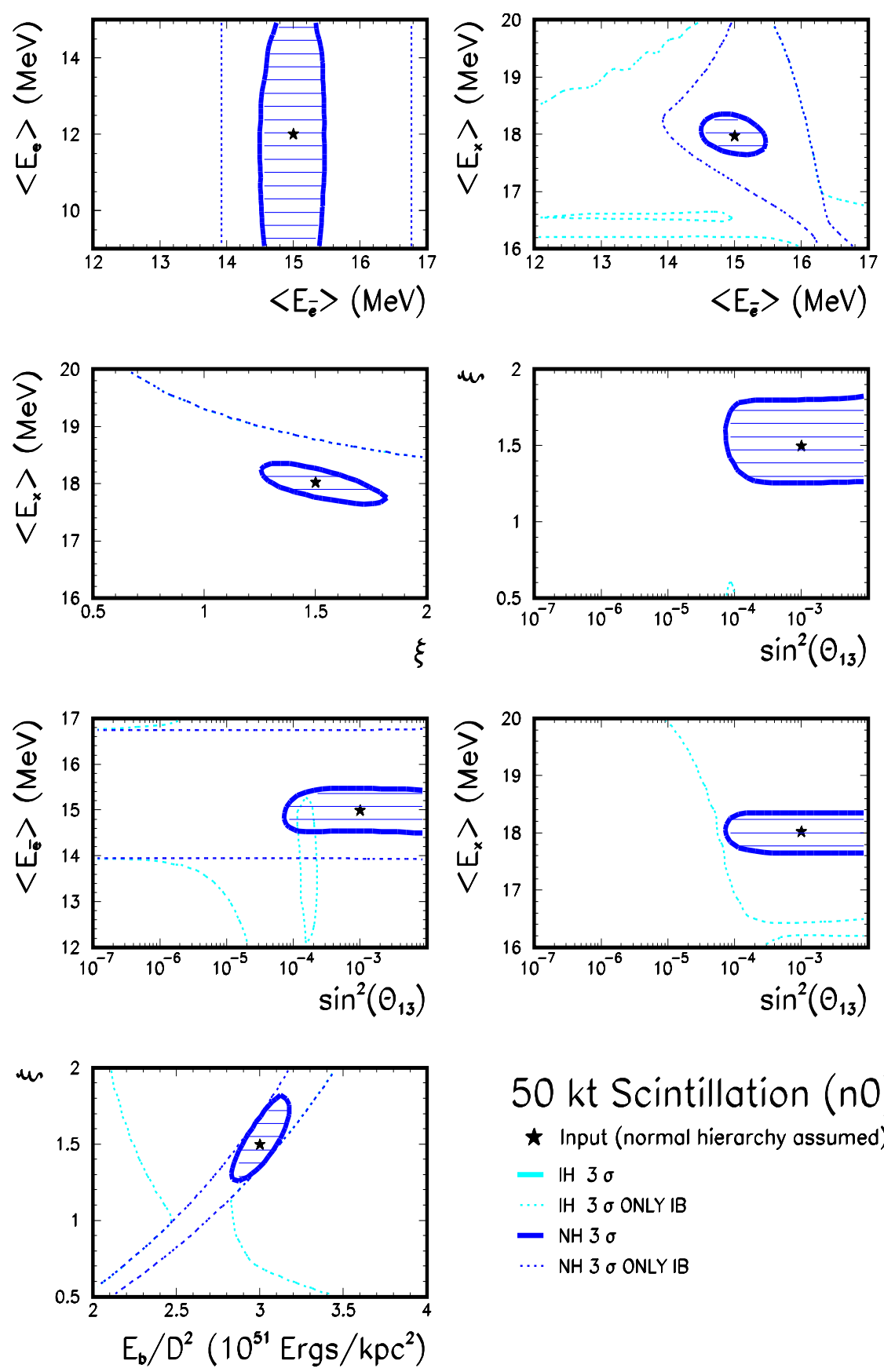

50 kt Scintillation (nO)

$\star$ Input (normal hierarchy assumed)

$-\mathrm{H} 3 \sigma$

… IH $3 \sigma O N L Y I B$

- $\mathrm{NH} 3 \sigma$

.... NH $3 \sigma$ ONLYIB

FIG. 13: Same as Fig. 11 but for the normal hierarchy and $\sin ^{2}\left(\theta_{13}\right)=10^{-3}\left(P_{H} \simeq 0\right)$. The inverted hierarchy is ruled out by more than $5 \sigma$, having a global $\chi_{\min }^{2}=60$ in the case of all six channels being present. 
will be extremely valuable. If several channels can be included in the analysis, the value of the SN parameters $\left\langle E_{x}\right\rangle,\left\langle E_{\bar{e}}\right\rangle$ (except for the LAr detector) and $E_{b} / D^{2}$ can be determined to a few $\%$ level in case a1. On the other hand, $\xi$ and $\left\langle E_{e}\right\rangle$ will be much less constrained by data (for point 1).

If $\sin ^{2} \theta_{13}$ is large, i.e. in the region where $P_{H} \simeq 0$ (cases i0 and $n 0$ ), all three experiments can determine the neutrino mass hierarchy. From Figure 6 and Figure 7 we clearly see that if $\theta_{13}$ is large, the neutrino mass hierarchy can be determined with a high confidence level independent of the true hierarchy, in a WaterC detector. Also the LAr and the Scintillation detectors can establish the hierarchy in the large $\theta_{13}$ region, as shown in Figs. 9, 10 and 12,13, although with less significance. It should be noted that, as we will discuss below, the establishment of the hierarchy depends on the parameter space assumed for the SN parameters, and in particular whether $\left\langle E_{\bar{e}}\right\rangle$ is allow to undertake the same value as $\left\langle E_{x}\right\rangle$.

In the case i0, the WaterC detector can determine the hierarchy and will give a lower limit on $\sin ^{2} \theta_{13}$, even if only the IB channel is available. For the LAr detector and the scenario with only the $\nu_{e} \mathrm{CC}$ channel being present, we see that the normal hierarchy can explain the i0 scenario. This is again evident from Fig.1 as in this case the information contained in the measurement is that $P_{e e} \simeq 0.3$, which occur for the normal hierarchy and small values of $\theta_{13}$ and for inverted hierarchy and any value of $\theta_{13}$. For the only IB case and the Scintillator detector, the measurement contain the information of the $\bar{\nu}_{e}$ flux with a temperature corresponding to the value of $\left\langle E_{x}\right\rangle$. This can also be fitted with a normal hierarchy and raising the value of $\left\langle E_{x}\right\rangle$ (giving about $30 \%$ of the flux) and $\left\langle E_{\bar{e}}\right\rangle$ (giving about $70 \%$ of the flux) such as to simulate a pure $\bar{\nu}_{e}$ flux of $18 \mathrm{MeV}$. The statistics of the WaterC detector is good enough to reject this case for the SN parameter space given in Eq16. If all channels are considered in the data analysis, the LAr and Scintillation detectors can produce a similar constraint on $\sin ^{2} \theta_{13}$ as the WaterC detector and the lower limit is given by $1-2 \times 10^{-4}$. For scenario i0 the $\bar{\nu}_{e}$ flux arriving at the Earth is identical to the original $\nu_{x}$ flux and thus in this case one looses much of the sensitivity to $\left\langle E_{\bar{e}}\right\rangle$. But, a slightly better sensitivity to $\left\langle E_{x}\right\rangle$ is obtained as compared to the a1 case, where a part of $F_{\bar{\nu}_{e}}$ is the original $\bar{\nu}_{e}$ flux. The LAr detector can measure $\left\langle E_{x}\right\rangle$ (even if only $\nu_{e}$ CC events are used) and $E_{b} / D^{2}$ to about $5 \%$, but will not be very sensitive to the other $\mathrm{SN}$ parameters. Also, the LENA-type detector, can determine $\left\langle E_{x}\right\rangle$ and $E_{b} / D^{2}$ to a few $\%$ with the help of all channels. The WaterC detector will be very sensitive to all SN parameters, except for $\left\langle E_{\bar{e}}\right\rangle$, if all channels contribute.

In the case $n 0$, even if only the main channels are available, $\left\langle E_{x}\right\rangle$ can be determined to a few $\%$ by the WaterC detector (see Fig:7). In this scenario there is basically no sensitivity to $\left\langle E_{e}\right\rangle$, as the $\nu_{e}$ flux is identical to the original $\nu_{x}$ flux. But, all the others SN parameters can be accessed with very good precision by the WaterC detector, if all channels take part in the 
analysis. The LAr setup can measure $\left\langle E_{x}\right\rangle$ to a few $\%$ and $\left\langle E_{\bar{e}}\right\rangle$ and $E_{b} / D^{2}$ to about $10 \%$ (see Fig (10). A LENA-type detector will have a sensitivity of about $5 \%$ for $\left\langle E_{x}\right\rangle,\left\langle E_{\bar{e}}\right\rangle$ and $E_{b} / D^{2}$ and about of $20 \%$ for $\xi$. Furthermore, if all channels are considered in the data analysis, the LAr and Scintillation detectors can again provide similar bounds on $\sin ^{2} \theta_{13}$ as the WaterC detector, giving a lower limit of $0.7-2 \times 10^{-4}$. If only the IB channel is available not even the WaterC detector can determine the hierarchy. As seen from Fig 1 the value of $P_{\bar{e} \bar{e}}$ of roughly 0.7 is also found for the inverted hierarchy and small values of $\theta_{13}$.

Let us shortly compare the sensitivity to the $\theta_{13}$ angle to that of other proposed future experiments. One expects the reactor experiment Double Chooz to reach down to $\sin ^{2} \theta_{13} \simeq$ $5 \times 10^{-3}$, and the next generation $\theta_{13}$ reactor experiment Daya-Bay to reach $\sin ^{2} \theta_{13} \simeq 2.5 \times$ $10^{-3}\left[55\right.$. The proposed novel technique which exploits the recoilless resonant absorption of $\bar{\nu}_{e}$ to measure $\sin ^{2} \theta_{13}$ in a short baseline experiment, may be able to reach similar sensitivity [56]. So to reach sensitivities to $\sin ^{2} \theta_{13} \lesssim 10^{-3}$ before a new galactic SN observation one would probably need beta-beams [57] or neutrino factories [58]. Correspondingly, the determination of a lower bound on $\theta_{13}$ of order $10^{-4}$ from a SN observation for the cases $\mathrm{i} 0$ and n0, can be of great importance.

In the following we shortly compare to the work in Ref.[19], where only the inverse beta decay channel at a WaterC detector was analyzed. In this paper only the case a1 is studied and the $\theta_{13}$ angle is not varied. This seems unlikely as there are no known experiment that can restrict $\theta_{13}$ to be smaller than $10^{-6}$, which would be necessary for getting independence of the exact value of the CHOOZ angle. Therefore, it is erroneously concluded in Ref. 19] that the value of $\xi$ can be well determined at HyperK with only the IB channel available. As can be seen from Fig 5 there is a degeneracy between $\theta_{13}$ and $\xi$, only broken by the addition of NC channels. This indetermination of $\xi$ in turn influence the measurement of $E_{b}$, as there is a degeneracy between $E_{b}$ and $\xi$ (see Fig.5 panel 9). Therefore, overall the allowed regions found in Ref.[19] are too restrictive as compared to our contours for the case of only IB.

Next we compare to the work in Ref.[18], where the WaterC detector with the inverse beta channel along with the CC-O was studied, although these were assumed inseparable. In addition the SNO detector was also analyzed, but as the fiducial mass is much smaller, this experiment does not add significantly to the statistics. The main sensitivity in Ref.[18] is to the $\bar{\nu}_{e}$ flux with only a minor sensitivity to the $\nu_{e}$ flux. In this paper it is concluded that the HyperK detector cannot determine the normal hierarchy even for large $\theta_{13}$. Clearly, we don't agree with this statement, as with the addition of the sub-dominant channels this is indeed possible. However, we see that if only the IB channel is available this conclusion is true. But, it must be remembered that the only-IB scenario is not a realistic one. We also note that for small $\theta_{13}$ the very precise determination of $\left\langle E_{\bar{e}}\right\rangle$ claimed in Ref.[18] is only possible for the case that normal 


\begin{tabular}{|c||ccc|ccc||}
\hline \multicolumn{1}{|c||}{} & \multicolumn{3}{c|}{ point 2 } & \multicolumn{3}{c||}{ point 3 } \\
& i0 & n0 & a1 & i0 & n0 & a1 \\
\hline \hline$\left\langle E_{e}\right\rangle \mathrm{IH}$ & $11.0-13.2$ & - & $11.1-13.4$ & $10.0-14.1$ & - & $<14.2$ \\
$\left\langle E_{e}\right\rangle \mathrm{NH}$ & - & $10.8-13.9$ & $10.5-13.0$ & $<13.8$ & NO & $<14.0$ \\
\hline$\left\langle E_{\bar{e}}\right\rangle \mathrm{IH}$ & $12.6-16.2$ & - & $14.5-15.2$ & NO & - & $14.4-15.2$ \\
$\left\langle E_{\bar{e}}\right\rangle \mathrm{NH}$ & - & $14.9-15.2$ & $14.9-15.2$ & $16.4-16.8$ & $14.7-15.2$ & $14.8-15.2$ \\
\hline$\left\langle E_{x}\right\rangle \mathrm{IH}$ & $17.9-18.1$ & - & $17.8-18.2$ & $16.4-16.6$ & - & $16.2-16.7$ \\
$\left\langle E_{x}\right\rangle \mathrm{NH}$ & - & $17.8-18.1$ & $17.7-18.1$ & $16.0-16.8$ & $17.3-17.7$ & $16.3-16.7$ \\
\hline$\xi \mathrm{IH}$ & $0.7-0.8$ & - & $0.6-0.8$ & $1.3-1.7$ & - & $>1.3$ \\
$\xi \mathrm{NH}$ & - & $0.7-0.8$ & $0.7-0.8$ & $0.7-0.9$ & $1.4-1.6$ & $1.3-1.7$ \\
\hline$E_{b} / D^{2} \mathrm{IH}$ & $2.9-3.1$ & - & $2.9-3.1$ & $2.8-3.2$ & - & $2.8-3.2$ \\
$E_{b} / D^{2} \mathrm{NH}$ & - & $2.9-3.1$ & $2.9-3.1$ & $2.9-3.2$ & $2.9-3.1$ & $2.8-3.2$ \\
\hline $\sin ^{2} \theta_{13} \mathrm{IH}$ & $>2 \cdot 10^{-4}$ & - & $<1 \cdot 10^{-5}$ & $>1 \cdot 10^{-4}$ & - & $<2 \cdot 10^{-5}$ \\
$\sin ^{2} \theta_{13} \mathrm{NH}$ & - & $>2 \cdot 10^{-4}<2 \cdot 10^{-5}$ & $>1 \cdot 10^{-5}$ & $>8 \cdot 10^{-5}$ & $<3 \cdot 10^{-5}$ \\
\hline Hier. det. & yes & yes & no & no & yes & no \\
\hline Hier. excl. & $>5 \sigma$ & $>5 \sigma$ & - & - & $>5 \sigma$ & - \\
\hline
\end{tabular}

TABLE V: The allowed parameter space at $3 \sigma$ CL (2 dof) for the points 2 and 3 of Table 1 by a 540 kt WaterC detector using all channels. The symbol '-' is used when there is no allowed area. In this case, we have written the confidence level with which a given hierarchy can be excluded. The symbol 'NO' is used when there are no restrictions on the parameter space given in Eq. (16). The energies are in $\mathrm{MeV}$ and $E_{b} / D^{2}$ in units of $10^{51} \mathrm{ergs} / \mathrm{kpc}^{2}$.

hierarchy has already been established.

In Tables V. VI and VII we show the allowed parameter space for the input point 2 of Table \. For point 2, we have chosen a smaller value of $\xi$, making the original $\nu_{e}$ and $\bar{\nu}_{e}$ fluxes twice as large as the original $\nu_{x}$ flux. There is not much difference between the results of point 1 and 2, except that the determination of $\left\langle E_{\bar{e}}\right\rangle$ and $\left\langle E_{e}\right\rangle$ are better for point 2. The accuracy of the determination of $\left\langle E_{x}\right\rangle$ has little dependence on the value of $\xi$, although it increases slowly for larger values of $\xi$.

In Tables Q V VI and VII we show the results for point 3 of Table I. For this point a weaker hierarchy between $\left\langle E_{x}\right\rangle$ and $\left\langle E_{\bar{e}}\right\rangle$ is considered, being about $10 \%$ for point 3 and about $20 \%$ for point 1. The main difference between these points, is the fact that in the case i0, the hierarchy can no longer be recognized. As mentioned above, the establishment of the hierarchy dependents on whether a value of $\left\langle E_{\bar{e}}\right\rangle$ equal to $\left\langle E_{x}\right\rangle$ is included in the scan over parameters. Had we enlarged the SN parameter space in Eq. (16) to include $\left\langle E_{\bar{e}}\right\rangle=18 \mathrm{MeV}$ we would find a very small allowed area for the WaterC detector for the normal hierarchy around the set of values: $\left\langle E_{\bar{e}}\right\rangle=18 \mathrm{MeV},\left\langle E_{e}\right\rangle \simeq 10 \mathrm{MeV},\left\langle E_{x}\right\rangle \simeq 18 \mathrm{MeV}, \xi \simeq 0.8, \sin ^{2} \theta_{13} \simeq 10^{-4}$ and $E_{b} / D^{2} \simeq 3.1 \times 10^{51} \mathrm{ergs} / \mathrm{kpc}^{2}$. It is not difficult to realize that this set of parameters produces 


\begin{tabular}{|c||ccc|ccc||}
\hline \multicolumn{1}{|c||}{} & \multicolumn{3}{c|}{ point 2 } & \multicolumn{3}{c||}{ point 3 } \\
& i0 & n0 & a1 & i0 & n0 & a1 \\
\hline \hline$\left\langle E_{e}\right\rangle \mathrm{IH}$ & $10.0-13.4$ & - & $<13.8$ & $<14.1$ & - & $<14.2$ \\
$\left\langle E_{e}\right\rangle \mathrm{NH}$ & - & $<14.6$ & $<13.8$ & $<12.7$ & NO & $<14.2$ \\
\hline$\left\langle E_{\bar{e}}\right\rangle \mathrm{IH}$ & $>13.2$ & - & $13.3-16.4$ & NO & - & $12.2-16.8$ \\
$\left\langle E_{\bar{e}}\right\rangle \mathrm{NH}$ & - & $13.8-16.2$ & $14.0-16.4$ & $>15.7$ & $13.2-16.8$ & $13.4-16.8$ \\
\hline$\left\langle E_{x}\right\rangle \mathrm{IH}$ & $17.4-18.6$ & - & $17.3-18.6$ & $16.1-17.0$ & - & $16.2-17.2$ \\
$\left\langle E_{x}\right\rangle \mathrm{NH}$ & - & $17.8-18.5$ & $17.4-18.6$ & $16.2-16.7$ & $16.3-16.9$ & $16.2-17.0$ \\
\hline$\xi \mathrm{IH}$ & $<1.4$ & - & $<1.5$ & $>0.7$ & - & $\mathrm{NO}$ \\
$\xi \mathrm{NH}$ & - & $0.6-1.1$ & $<1.3$ & $0.7-1.3$ & $>1.0$ & $>0.7$ \\
\hline$E_{b} / D^{2} \mathrm{IH}$ & $2.8-3.2$ & - & $2.8-3.2$ & $2.8-3.2$ & - & $2.8-3.1$ \\
$E_{b} / D^{2} \mathrm{NH}$ & - & $2.7-3.3$ & $2.8-3.3$ & $2.9-3.4$ & $2.7-3.3$ & $2.8-3.2$ \\
\hline $\sin ^{2} \theta_{13} \mathrm{IH}$ & $>6 \cdot 10^{-5}$ & - & $<5 \cdot 10^{-5}$ & $>6 \cdot 10^{-5}$ & - & $<2 \cdot 10^{-5}$ \\
$\sin ^{2} \theta_{13} \mathrm{NH}$ & - & $>6 \cdot 10^{-5}$ & $<2 \cdot 10^{-5}$ & $1-8 \cdot 10^{-5}$ & $>6 \cdot 10^{-5}$ & $<4 \cdot 10^{-5}$ \\
\hline Hier. det. & yes & yes & no & no & yes & no \\
\hline Hier. excl. & $>5 \sigma$ & $3 \sigma$ & - & - & $>5 \sigma$ & - \\
\hline
\end{tabular}

TABLE VI: Same as Table V but for a $100 \mathrm{kt}$ LAr detector.

total number of events in each channel only slightly different from the i0 case. Improving the energy resolution of the detector does not help much. For point 1 the normal hierarchy is excluded at 95\% CL and for point 3 by less than $1 \sigma$ for the WaterC detector. Nevertheless, as the allowed region for the normal hierarchy in this case is very small and occurring for the $\bar{\nu}_{e}$ and $\nu_{x}$ fluxes having equal temperatures, one would naturally have a strong hint that the true hierarchy is indeed the inverted hierarchy. An analog discussion could be performed for the case n0. However, as this would require all three average energies to be almost equal, we will refrain from this discussion, as it is physically very improbable.

Next, we would like to discuss how the detection of Earth matter effects and shock wave effects can help to pin down the hierarchy in the i0 scenario when allowing the $\bar{\nu}_{e}$ and $\nu_{x}$ fluxes to have identical temperatures. Let us shortly review the facts about Earth matter effects for SN neutrinos [23, 24]. For neutrinos that traverse the Earth mantle (and core) a modulation with known frequencies of the neutrino energy spectra may occur, depending on the hierarchy and the value of $\theta_{13}$. If the hierarchy is normal (inverted), the Earth matter effect for neutrinos (anti-neutrinos) depend on the value of $P_{H}$. In both cases, the strength of the modulation will be proportional to the difference in the original $\nu_{x}$ and $\nu_{e}\left(\nu_{\bar{e}}\right)$ fluxes and the value of $P_{H}$. Therefore, in the case of normal hierarchy and large values of $\left.\theta_{13}\left(P_{H} \simeq 0\right)\right)$ there should be a modulation of the anti-neutrino energy-spectra unless $\left\langle E_{\bar{e}}\right\rangle=\left\langle E_{x}\right\rangle$ and no modulation of the neutrino spectra. Similarly, in the case i0 there should be a modulation of the neutrino 


\begin{tabular}{|c|c|c|c|c|c|c|}
\hline & \multicolumn{3}{|c|}{ point 2} & \multicolumn{3}{|c|}{ point 3} \\
\hline & i0 & n0 & a1 & i0 & n0 & a1 \\
\hline$\left\langle E_{e}\right\rangle \mathrm{IH}$ & $10.1-14.2$ & - & $<14.8$ & $\mathrm{NO}$ & - & $\mathrm{NO}$ \\
\hline$\left\langle E_{e}\right\rangle \mathrm{NH}$ & - & $<14.3$ & $<14.7$ & $\mathrm{NO}$ & $\mathrm{NO}$ & NO \\
\hline$\left\langle E_{\bar{e}}\right\rangle \mathrm{IH}$ & $12.2-16.8$ & - & $13.6-15.3$ & $\mathrm{NO}$ & - & $13.6-15.6$ \\
\hline$\left\langle E_{\bar{e}}\right\rangle \mathrm{NH}$ & - & $14.6-15.3$ & $14.6-15.3$ & $\mathrm{NO}$ & $14.5-15.5$ & $14.5-15.5$ \\
\hline$\left\langle E_{x}\right\rangle \mathrm{IH}$ & $17.7-18.3$ & - & $17.5-18.7$ & $16.1-17.0$ & - & $16.0-16.9$ \\
\hline$\left\langle E_{x}\right\rangle \mathrm{NH}$ & - & $17.6-18.4$ & $17.4-18.4$ & $15.9-16.9$ & $16.2-16.8$ & 16.1-16.9 \\
\hline$\xi \mathrm{IH}$ & $0.6-1.0$ & - & $<0.8$ & $<0.8$ or $>1.0$ & - & $>1.1$ \\
\hline$\xi \mathrm{NH}$ & - & $0.6-0.9$ & $<0.9$ & $0.6-1.0$ & $1.2-1.9$ & $1.1-1.9$ \\
\hline$E_{b} / D^{2} \mathrm{IH}$ & $2.7-3.3$ & - & $2.8-3.3$ & $2.8-3.3$ & - & $2.8-3.2$ \\
\hline$E_{b} / D^{2} \mathrm{NH}$ & - & $2.8-3.2$ & $2.8-3.2>10^{-4}$ & $2.8-3.3$ & $2.8-3.2$ & 2.8-3.2 \\
\hline $\sin ^{2} \theta_{13} \mathrm{IH}$ & $>1 \cdot 10^{-4}$ & - & $<2 \cdot 10^{-5}$ & $<10^{-5}$ or $>10^{-4}$ & - & $<2 \cdot 10^{-5}$ \\
\hline $\sin ^{2} \theta_{13} \mathrm{NH}$ & - & $>6 \cdot 10^{-5}$ & $<4 \cdot 10^{-5}$ & $\mathrm{NO}$ & $>5 \cdot 10^{-5}$ & $<5 \cdot 10^{-5}$ \\
\hline Hier. det. & yes & yes & no & no & yes & no \\
\hline Hier. excl. & $>5 \sigma$ & $>5 \sigma$ & - & - & $>5 \sigma$ & - \\
\hline
\end{tabular}

TABLE VII: Same as Table $\mathrm{V}$ but for a $50 \mathrm{kt}$ Scintillation detector.

energy-spectra unless $\left\langle E_{e}\right\rangle=\left\langle E_{x}\right\rangle$ and there should be no modulation of the anti-neutrino spectra. This can clearly help to distinguish the hierarchy in case i0 for point 1 , as we do have a hierarchy between the neutrino and $\nu_{x}$ average energies. With detectors shielded and unshielded by Earth, telling us that there are Earth matter effects in the neutrino channel and not in the anti-neutrino channel, the case i0 is clearly established. The expectation for point 1 , is a maximum difference of the neutrino flux arriving at Earth and the flux after traversing part of the Earth matter of about 20\%, occurring for a neutrino energy of roughly $60 \mathrm{MeV}$. Obviously for point 3 it will be much more difficult to observe and determine the Earth matter effects, as the weak hierarchy between $\left\langle E_{\bar{e}}\right\rangle$ and $\left\langle E_{x}\right\rangle$ will make the overall strength of the Earth matter effects smaller. Furthermore, shock-wave effects [30] may also help to identify the true hierarchy. These effects can be seen in the adiabatic region of the high $(\mathrm{H})$ resonance, i.e. for large values of $\theta_{13}$. In the case of inverted hierarchy a dip in the value of $\left\langle E_{\bar{e}}\right\rangle$ as a function of time is expected, whereas for normal hierarchy the dip is expected for $\left\langle E_{e}\right\rangle$. Therefore, an observation of shock wave effects in the anti-neutrino channel will point toward the inverted hierarchy. Once again the amplitude of this effects decrease as the $\nu_{e} / \bar{\nu}_{e}$ and $\nu_{x}$ temperatures becomes closer, making it difficult to pin down the hierarchy for point 1 . In conclusion, the hierarchy for case i0 is likely to be established with the detection of the SN neutrinos at Earth alone. The confidence level with which this can be done increase as the hierarchy between the $\bar{\nu}_{e}$ and $\nu_{x}$ temperatures increase. With the complementary information on Earth matter effects 
and shock wave effects it is very likely that the hierarchy can be undoubtedly established for point 1 .

Summarizing, the values of some or even all of the SN parameters as well as the unknown neutrino ones, might be determined simultaneously, in most cases. The values of $\xi,\left\langle E_{\bar{e}}\right\rangle$ and $\left\langle E_{e}\right\rangle$ are however difficult to determine with a high precision. Overall, there is not much difference in the performance of all three detectors. The WaterC can access most SN parameters with a higher accuracy, but this is basically due to the larger mass and hence statistics. The LENA-type detector is performing slightly better than LAr (except for the determination of $\left.\left\langle E_{e}\right\rangle\right)$ for the cases a1 and i0. For the n0 case they are almost equally good, with LAr determining $\left\langle E_{e}\right\rangle,\left\langle E_{x}\right\rangle$ better and LENA doing a better job in determining $\xi$. Therefore, generally the two detectors have similar performances, although the mass of the LAr detector is twice as big as that of LENA. This difference can be understood as the LAr detector has a rather weak sensitivity to the pure anti-electron neutrino flux. The LENA-type detector, on the other hand, has a good distribution between sensitivity to pure $\nu_{e}$, pure $\bar{\nu}_{e}$ and the total neutrino flux.

\section{CONCLUSIONS}

We have studied the prospects for extracting, simultaneously SN parameters and neutrino oscillation parameters from the measurements of neutrinos from the cooling phase of a galactic supernova in three different detectors: a megaton-scale water Cherenkov, a 100 kton liquid Argon and a 50 kton scintillation detector.

In our analysis we have varied a total of seven parameters, five SN parameters: the average energies $\left\langle E_{e}\right\rangle,\left\langle E_{\bar{e}}\right\rangle$ and $\left\langle E_{x}\right\rangle$, the ratio of the luminosities $\xi$ and the overall normalization of the fluxes $E_{b} / D^{2}$; two neutrino oscillation parameters: the angle $\theta_{13}$ and the neutrino mass hierarchy. Since we considered perfect detectors, with 100\% efficiencies, our analysis must be viewed as an estimation of the maximal performance of each experimental setup. We do not include Earth matter effects or shock-wave effects in our calculations but briefly discuss their possible implications.

Our main results are summarized in Figs. 5,7, 8,10 and 11,13, for the WaterC, LAr and Scintillator detectors, respectively. We have found that SN parameters, as well as the unknown neutrino ones, can be determined simultaneously, in most cases. Comparing the three detectors, there is not much difference in their overall performance. However, the WaterC detector can access most SN parameters with higher accuracy (a few \% in some cases), but this is basically due to its larger mass and statistics.

All of the studied detectors have the possibility to determine the neutrino mass hierarchy if 
$\sin ^{2}\left(\theta_{13}\right) \gtrsim 10^{-4}$ and the hierarchy of the average energies is stronger than about $20 \%$. They can at the same time determine some of the SN parameters quite well and put strong bounds on the value of $\theta_{13}$. The average energy of the $\nu_{\mu}$ and $\nu_{\tau}$ species can be determined with an accuracy better than $5 \%$ in most of the parameter space suggested by SN simulations.

Although the detection of several separable channels measuring different combinations of $\mathrm{CC}$ and NC processes is crucial for the determination of $\theta_{13}$ and the hierarchy, there are cases where some SN parameters can be determined rather well even when only the main CC detection channel is available.

\section{APPENDIX A: Allowed regions for detectors at non-optimal performances}

In this appendix we show contours for a few cases, where we assume that some channels are either non-separable from other channels or not available at all.

We investigate the case of a WaterC detector where the inverse beta decay channel cannot be separated from the CC on Oxygen. We have chosen this scenario, as indeed this separation might be difficult if gadolinium will not be added (in which case the neutron is not detectable), since both detection processes are almost isotropic. We show the a1 case in Fig 14. Comparing to the case of all channels being separable in Fig 5 (solid lines) we see that the biggest difference is the determination of $\sin ^{2} \theta_{13}$ for the normal hierarchy allowed region. The upper bound on $\sin ^{2} \theta_{13}$ increases from $2 \times 10^{-5}$ to $5 \times 10^{-5}$. Besides this the other parameters have very similar restrictions. Moreover, the contours for inverted hierarchy are almost identical to the ones in Fig 5 (solid lines). This is expected as for the inverted hierarchy the inverse beta decay channel dominates and therefore whether or not it can be separated from the CC-O does not have a large effect. This is also the reason that the scenario i0 does not change much if the IB and CC-O cannot be separated. The only visible difference is a slightly worse determination of $\left\langle E_{e}\right\rangle$, but this variable can not be determined very well in either scenarios. For the scenario n0, the lower bound on $\sin ^{2} \theta_{13}$ jumps from $1 \times 10^{-4}$ to $6 \times 10^{-5}$. Overall, the separation of the IB and $\mathrm{CC}-\mathrm{O}$ channel have little impact on the detector performance, besides a less restrictive bound on the CHOOZ angle.

Next we study the LAr detector in the case that the neutral current on Argon is not available. This NC channel is not accompanied by the detection of the positron or electron and might therefore be difficult to trigger, especially if the detector is not well shielded. We show the case where the input scenario is n0 in Fig 15. Comparing Fig 15 to Fig 10 (solid lines) we see that again the biggest impact is a worsening of the determination of $\sin ^{2} \theta_{13}$. In this case the impact is severe, lowering the upper bound from $10^{-4}$ to $7 \times 10^{-7}$. Other parameters are only mildly affected. For the case i0, the normal hierarchy cannot be ruled out with the 3 channel scenario, 

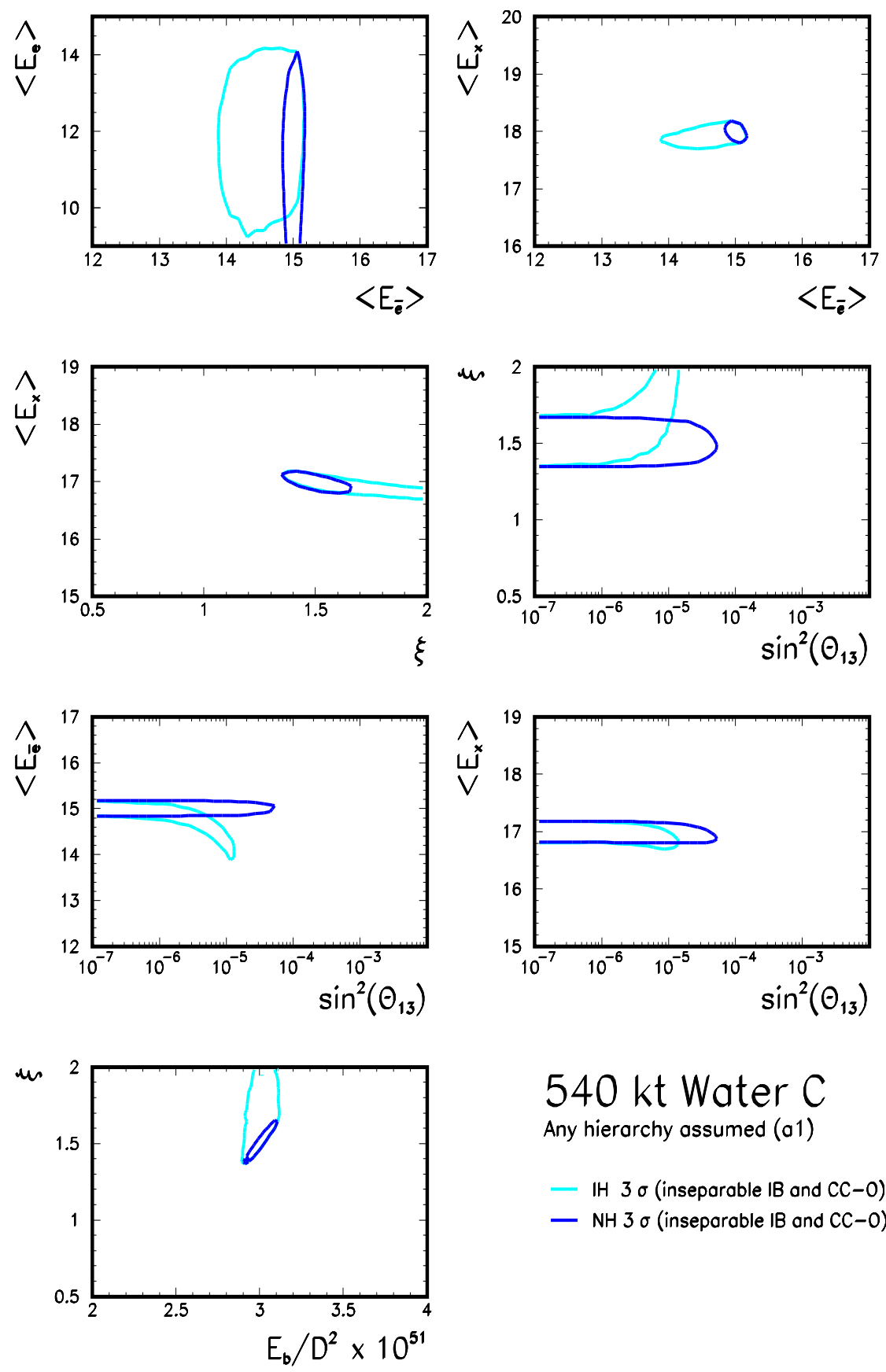

\section{$540 \mathrm{kt}$ Water C} Any hierarchy assumed (a1)

- $\mathrm{IH} 3 \sigma$ (inseparable $\mathrm{IB}$ and $\mathrm{CC}-\mathrm{O}$ )

- $\mathrm{NH} 3 \sigma$ (inseparable $\mathrm{IB}$ and $\mathrm{CC}-0$ )

FIG. 14: Sensitivity of a 540 kton WaterC detector assuming true SN parameters as in point 1 of Table I, for any hierarchy and $\sin ^{2}\left(\theta_{13}\right)=10^{-6}\left(P_{H} \simeq 1\right)$. We show $3 \sigma$ CL contours (2 dof) using all 4 detection processes (IB + CC-O + ELAS + NC-O), but assuming that the IB and CC-O cannot be distinguished. The contours for the normal hierarchy $(\mathrm{NH})$ is marked by the dark (red) and for the inverted hierarchy (IH) is marked by the light (cyan) solid line. 

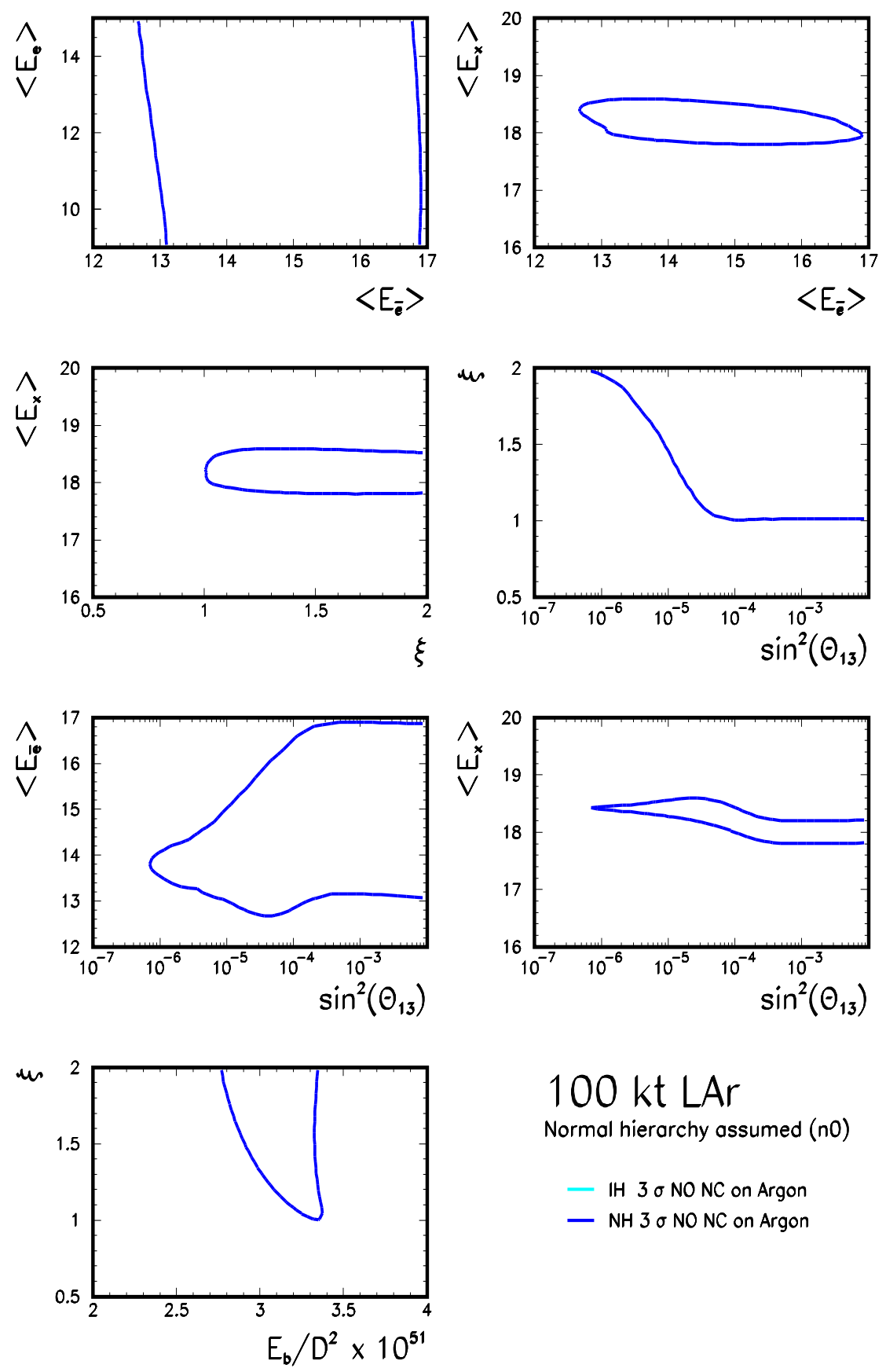

- $\mathrm{H} 3 \sigma$ NONC on Argon

- NH $3 \sigma$ NO NC on Argon

FIG. 15: Sensitivity of a 100 kton LAr detector assuming true SN parameters as in point 1 of Table I. for normal hierarchy and $\sin ^{2}\left(\theta_{13}\right)=10^{-3}\left(P_{H} \simeq 0\right)$. We show $3 \sigma$ CL contours (2 dof) using 3 channels $\left(\nu_{e} \mathrm{CC}+\bar{\nu}_{e} \mathrm{CC}+\mathrm{ELAS}\right)$, assuming that the $\mathrm{NC}$ on Argon is not available. The contours for normal hierarchy is marked by the dark (red) and for inverted hierarchy is marked by the light (cyan) solid line. The inverted hierarchy is ruled out by more than $5 \sigma$. 

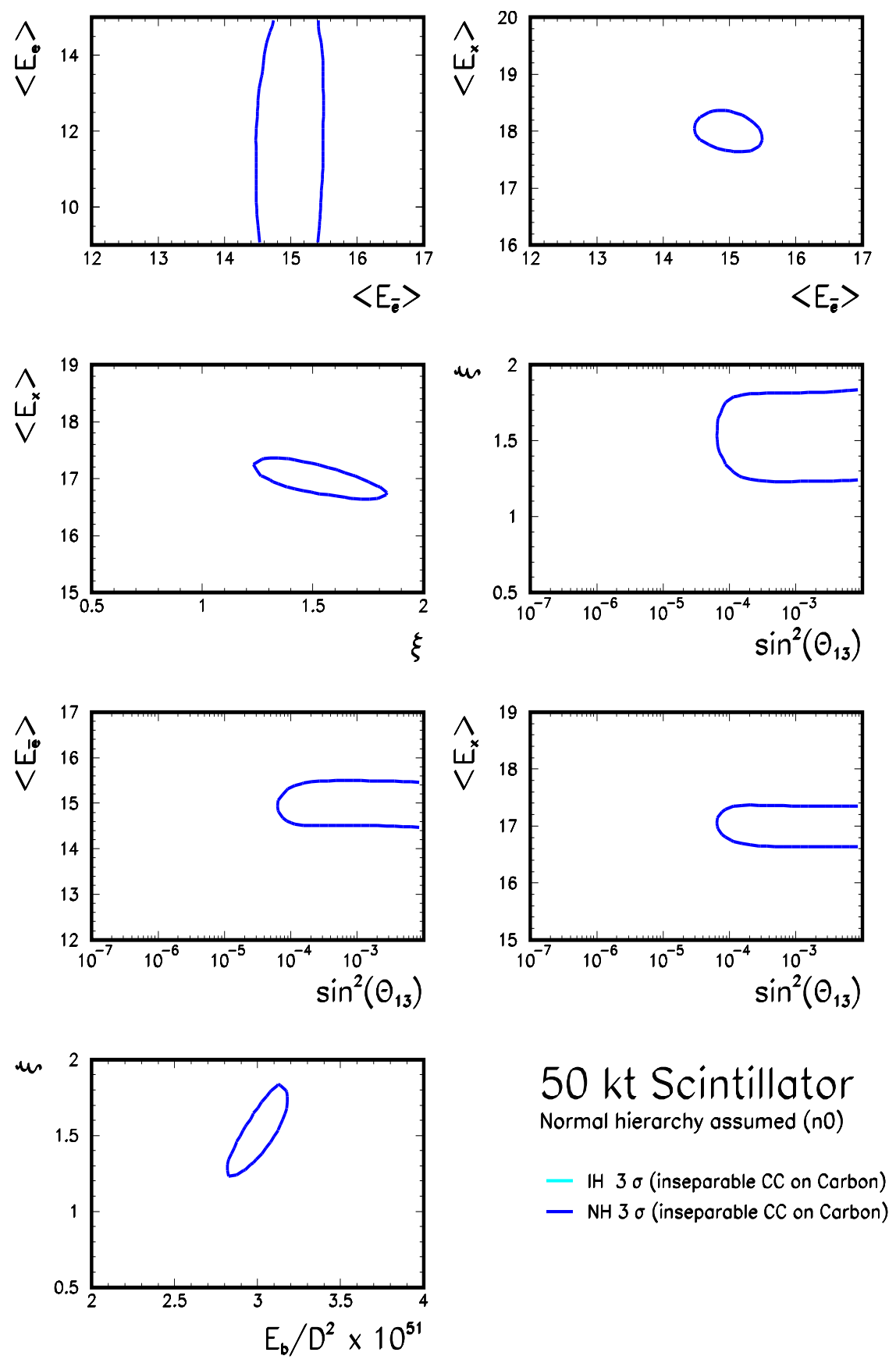

FIG. 16: Sensitivity of a 50 kton Scintillation detector assuming true SN parameters as in point 1 of Table @, for normal hierarchy and $\sin ^{2}\left(\theta_{13}\right)=10^{-3}\left(P_{H} \simeq 0\right)$. We show $3 \sigma$ CL contours (2 dof) using all 6 detection processes (IB $+\nu_{e} \mathrm{CC}+\bar{\nu}_{e} \mathrm{CC}+\nu-p+\mathrm{NC}+$ ELAS) but assuming that the $\nu_{e} \mathrm{CC}$ and $\bar{\nu}_{e} \mathrm{CC}$ cannot be distinguished. The contours for normal hierarchy is marked by the dark (red) and for inverted hierarchy is marked by the light (cyan) solid line. The inverted hierarchy is ruled out by more than $5 \sigma$. 
as a small 'fake' region around $\left\langle E_{\bar{e}}\right\rangle=17 \mathrm{MeV}$ is allowed for small values of $\sin ^{2} \theta_{13}$. This also leaves $\sin ^{2} \theta_{13}$ unrestricted for the inverted hierarchy, unless one constrains $\left\langle E_{\bar{e}}\right\rangle$ to be less than about $16.5 \mathrm{MeV}$, in which case the restriction is roughly as the case with 4 channels. For the input scenario a1, again the biggest impact is a drop in the upper bound on $\sin ^{2} \theta_{13}$ from $2 \times 10^{-5}$ to $6 \times 10^{-5}$. We conclude that the $\mathrm{NC}$ channel on Argon is an important factor for determining the neutrino parameters: the hierarchy and in particular the value of the CHOOZ angle.

Finally we investigate the Scintillator detector assuming that the neutrino and anti-neutrino $\mathrm{CC}$ reactions on Carbon cannot be separated. We show the case of the input scenario n0 in Fig.16. Comparing Fig 16 to Fig.13 (solid lines) we see that there is hardly any visible difference. This is also valid for the two other cases (a1 and i0). The reason for this is that the dominant inverse beta decay channel is still a separate channel and thus gives good restrictions on the $\bar{\nu}_{e}$ flux, which in turn makes a de facto separation of the $\nu_{e}$-CC and $\bar{\nu}_{e}$-CC channels when doing the fitting. We conclude that the separation of the $\mathrm{CC}$ channels on Carbon only have a minor impact on the performance of the Scintillator detector.

\section{Acknowledgments}

We thank Fundação de Amparo à Pesquisa do Estado de São Paulo (FAPESP) and Conselho Nacional de Ciência e Tecnologia (CNPq) for financial support.

\section{References}

[1] K. Hirata et al. [KAMIOKANDE-II Collaboration], Phys. Rev. Lett. 58, 1490 (1987).

[2] R. M. Bionta et al., Phys. Rev. Lett. 58, 1494 (1987).

[3] For a review see, K. Scholberg, Nucl. Phys. Proc. Suppl. 91, 331 (2000) arXiv:hep-ex/0008044.

[4] H. A. Bethe and J. R. Wilson, Astrophys. J. 295, 14 (1985).

[5] R. Buras, H. T. Janka, M. Rampp and K. Kifonidis, arXiv:astro-ph/0512189;

G. G. Raffelt, M. T. Keil, R. Buras, H. T. Janka and M. Rampp, arXiv:astro-ph/0303226; M. T. Keil, G. G. Raffelt and H. T. Janka, Astrophys. J. 590, 971 (2003) arXiv:astro-ph/0208035.

[6] T. Totani, K. Sato, H. E. Dalhed and J. R. Wilson, Astrophys. J. 496, 216 (1998) arXiv:astro-ph/9710203. 
[7] Y. Ashie Y et al. (Super-Kamiokande Collaboration), Phys. Rev. D 71, 112005 (2005).

[8] B. T. Cleveland et al., Astrophys. J. 496, 505 (1998); C. Cattadori, Results from radiochemical solar neutrino experiments, talk given at XXIst International Conference on Neutrino Physics and Astrophysics (NU2004), Paris, June 14-19, 2004; W. Hampel et al. (GALLEX Collaboration), GALLEX collaboration, Phys. Lett. B 447, 127 (1999). S. Fukuda et al. (Super-Kamiokande Collaboration), Phys. Rev. Lett. 86, 5651 (2001); Q. R. Ahmad et al. (SNO Collaboration), Phys. Rev. Lett. 87, 071301 (2001); Phys. Rev. Lett. 89, 011302 (2002); S. N. Ahmed et al. (SNO Collaboration), Phys. Rev. Lett. 92, 181301 (2004); S. B. Aharmim et al. (SNO Collaboration), arXiv:nucl-ex/0502021.

[9] T. Araki et al. (KamLAND Collaboration), Phys. Rev. Lett. 94, 081801 (2005); K. Eguchi et al. (KamLAND Collaboration), Phys. Rev. Lett. 90, 021802 (2003).

[10] E. Aliu et al. (K2K Collaboration), Phys. Rev. Lett. 94, 081802 (2005).

[11] N. Tagg (MINOS Collaboration), Proc. 4th Flavor Physics and CP Violation Conference (Vancouver) eConf C060409, 019 (2006) arXiv:hep-ex/0605058].

[12] D. N. Spergel et al., arXiv:astro-ph/0603449.

[13] M. Apolloni et al. (CHOOZ Collaboration), Phys. Lett. B420, 397 (1998); Eur. Phys. J. C 27, 331 (2003).

[14] A. S. Dighe and A. Y. Smirnov, Phys. Rev. D 62, 033007 (2000) arXiv:hep-ph/9907423.

[15] C. Lunardini and A. Y. Smirnov, JCAP 0306, 009 (2003) arXiv:hep-ph/0302033.

[16] K. Takahashi and K. Sato, Nucl. Phys. A 718, 455 (2003).

[17] A. Rubbia, Nucl. Phys. Proc. Suppl. 147, 103 (2005) arXiv:hep-ph/0412230.

[18] V. Barger, D. Marfatia and B. P. Wood, Phys. Lett. B 547, 37 (2002) arXiv:hep-ph/0112125.

[19] H. Minakata, H. Nunokawa, R. Tomas and J. W. F. Valle, Phys. Lett. B 542, 239 (2002) arXiv:hep-ph/0112160.

[20] I. Gil-Botella and A. Rubbia, JCAP 0408, 001 (2004) arXiv:hep-ph/0404151.

[21] E. Nardi and J. I. Zuluaga, Nucl. Phys. B 731, 140 (2005) arXiv:hep-ph/0412104. 
[22] J. Arafune, M. Fukugita, T. Yanagida and M. Yoshimura, Phys. Rev. Lett. 59, 1864 (1987).

[23] C. Lunardini and A. Y. Smirnov, Nucl. Phys. B 616, 307 (2001) arXiv:hep-ph/0106149.

[24] A. S. Dighe, M. Kachelriess, G. G. Raffelt and R. Tomas, JCAP 0401, 004 (2004) arXiv:hep-ph/0311172.

[25] A. Mirizzi, G. G. Raffelt and P. D. Serpico, JCAP 0605, 012 (2006) arXiv:astro-ph/0604300.

[26] X. H. Guo and B. L. Young, Phys. Rev. D 73, 093003 (2006) arXiv:hep-ph/0605122.

[27] K. Takahashi, M. Watanabe, K. Sato and T. Totani, Phys. Rev. D 64, 093004 (2001) arXiv:hep-ph/0105204.

[28] V. Barger, P. Huber and D. Marfatia, Phys. Lett. B 617, 167 (2005) arXiv:hep-ph/0501184.

[29] R. C. Schirato and G. M. Fuller, arXiv:astro-ph/0205390.

[30] R. Tomas, M. Kachelriess, G. Raffelt, A. Dighe, H. T. Janka and L. Scheck, JCAP 0409, 015 (2004) arXiv:astro-ph/0407132.

[31] G. L. Fogli, E. Lisi, A. Mirizzi and D. Montanino, JCAP 0504, 002 (2005) arXiv:hep-ph/0412046.

[32] G. L. Fogli, E. Lisi, A. Mirizzi and D. Montanino, JCAP 0606, 012 (2006) arXiv:hep-ph/0603033.

[33] S. Choubey, N. P. Harries and G. G. Ross, Phys. Rev. D 74, 053010 (2006) arXiv:hep-ph/0605255.

[34] S. H. Chiu and T. K. Kuo, Phys. Rev. D 73, 033007 (2006) [Erratum-ibid. D 73, 059901 (2006)].

[35] H. Duan, G. M. Fuller, J. Carlson and Y. Z. Qian, arXiv:astro-ph/0608050. H. Duan, G. M. Fuller, J. Carlson and Y. Z. Qian, Phys. Rev. D 74, 105014 (2006) arXiv:astro-ph/0606616.

[36] R. Tomas, D. Semikoz, G. G. Raffelt, M. Kachelriess and A. S. Dighe, Phys. Rev. D 68, 093013 (2003) arXiv:hep-ph/0307050. 
[37] Y. Itow et al., arXiv:hep-ex/0106019.

[38] C. K. Jung, AIP Conf. Proc. 533, 29 (2000) arXiv:hep-ex/0005046].

[39] A. de Bellefon et al., arXiv:hep-ex/0607026.

[40] P. Vogel and J. F. Beacom, Phys. Rev. D 60, 053003 (1999) arXiv:hep-ph/9903554.

[41] E. Kolbe, K. Langanke and P. Vogel, Phys. Rev. D 66, 013007 (2002).

W. C. Haxton, Phys. Rev. D 36, 2283 (1987).

[42] K. Langanke, P. Vogel and E. Kolbe, Phys. Rev. Lett. 76, 2629 (1996) arXiv:nucl-th/9511032.

[43] J. F. Beacom and M. R. Vagins, Phys. Rev. Lett. 93, 171101 (2004) arXiv:hep-ph/0309300.

[44] D. B. Cline, F. Sergiampietri, J. G. Learned and K. McDonald, Nucl. Instrum. Meth. A 503, 136 (2003) arXiv:astro-ph/0105442.

[45] A. Ereditato and A. Rubbia, Nucl. Phys. Proc. Suppl. 154, 163 (2006) arXiv:hep-ph/0509022. A. Rubbia, arXiv:hep-ph/0402110.

[46] D. Fargion, PoS HEP2005, 180 (2006) arXiv:astro-ph/0512181.

[47] A. Bueno, I. Gil-Botella and A. Rubbia, arXiv:hep-ph/0307222.

[48] I. Gil-Botella and A. Rubbia, JCAP 0310, 009 (2003) arXiv:hep-ph/0307244.

[49] E. Kolbe, K. Langanke, G. Martinez-Pinedo and P. Vogel, J. Phys. G 29, 2569 (2003) arXiv:nucl-th/0311022.

[50] L. Oberauer, F. von Feilitzsch, and W. Potzel, Nucl. Phys. Proc. Suppl. 138, 108 (2005).

[51] T. Marrodan Undagoitia, F. von Feilitzsch, M. Goder-Neff, K. A. Hochmuth, L. Oberauer, W. Potzel and M. Wurm, Prog. Part. Nucl. Phys. 57, 283 (2006) [J. Phys. Conf. Ser. 39, 278 (2006)] arXiv:hep-ph/0605229.

[52] L. Cadonati, F. P. Calaprice and M. C. Chen, Astropart. Phys. 16, 361 (2002) arXiv:hep-ph/0012082. A. Bandyopadhyay, S. Choubey, S. Goswami and K. Kar, arXiv:hep-ph/0312315. N. Y. Agafonova et al., arXiv:hep-ph/0609305. 
[53] J. F. Beacom, W. M. Farr and P. Vogel, Phys. Rev. D 66, 033001 (2002) arXiv:hep-ph/0205220.

[54] J. Engel, E. Kolbe, K. Langanke and P. Vogel, Phys. Rev. C 54, 2740 (1996) arXiv:nucl-th/9606031]; E. Kolbe, F. K. Thielemann, K. Langanke and P. Vogel, Phys. Rev. C 52, 3437 (1995); E. Kolbe, K. Langanke and S. Krewald, Phys. Rev. C 49, 1122 (1994).

[55] K. Anderson et al., White Paper Report on Using Nuclear Reactors to Search for a Value of $\theta_{13}$, arXiv:hep-ex/0402041.

[56] H. Minakata and S. Uchinami, New J. Phys. 8, 143 (2006) arXiv:hep-ph/0602046].

[57] J. Burguet-Castell, D. Casper, J. J. Gomez-Cadenas, P. Hernandez and F. Sanchez, Nucl. Phys. B 695, 217 (2004) arXiv:hep-ph/0312068; S. K. Agarwalla, A. Raychaudhuri and A. Samanta, Phys. Lett. B 629, 33 (2005) arXiv:hep-ph/0505015].

[58] P. Huber, M. Lindner, M. Rolinec and W. Winter, Phys. Rev. D 74, 073003 (2006) arXiv:hep-ph/0606119. J. T. Peltoniemi and J. Sarkamo, Nucl. Phys. Proc. Suppl. 155, $201(2006)$. 